\title{
Information Theory to Assess Relations Between Energy and Structure of the U.S. Economy Over Time
}

\author{
Carey W. King ${ }^{1}$
}

Received: 14 May 2016/ Accepted: 26 September 2016/Published online: 31 October 2016

(c) Springer International Publishing Switzerland 2016

\begin{abstract}
This paper describes the changing structure of the United States' (U.S.) domestic economy by applying information theory-based metrics to the U.S. input-output (I-O) tables from 1947 to 2012. Here the I-O tables are an economic network where the sectors are the nodes. The value of these metrics is that they describe the balance or trade-off between efficiency and redundancy of network flows as well as equality and hierarchy of flows through nodes in a network. I relate these metrics to the U.S. gross power consumption and annual intermediate spending by the food and energy sectors, the latter being a proxy for the inverse of the net power ratio (or net energy) of the economy, to test hypotheses of energy-economy structural linkages. The results of this paper show that increasing gross power consumption, as well as a decreasing share of intermediate expenditures of the food and energy sectors, correlates with increased distribution of money among economic sectors, and vice versa. The information theory metrics indicate two time periods at which major structural shifts occurred. The first was between 1967 and 1972, and the second was around the turn of the twenty-first century when food and energy expenditures no longer continued to decrease after 2002. In response to the latter, it is clear that the U.S. economy did trade off structural reserves (e.g., decreasing metrics of conditional entropy, redundancy, and
\end{abstract}

Electronic supplementary material The online version of this article (doi:10.1007/s41247-016-0011-y) contains supplementary material, which is available to authorized users.

Carey W. King

careyking@mail.utexas.edu

1 Research Scientist and Assistant Director Energy Institute, The University of Texas at Austin, 2304 Whitis Ave., C2400, Austin, TX 78712, USA equality) for structural efficiency (e.g., increasing metrics of efficiency, mutual constraint, and hierarchy) after food and energy expenditures increased post-2002. I also test the structural trends with increasingly simpler (e.g., fewer sectors) representations of the $\mathrm{I}-\mathrm{O}$ tables, and the results are more consistent for $\mathrm{I}-\mathrm{O}$ representations that account for inputs and outputs (e.g., value added and gross domestic product) rather than only the intermediate transactions among sectors. The findings of this paper have important implications for economic modeling in at least two ways. First, the paper helps explain how fundamental shifts in resources costs relate to economic structure and economic growth. Second, the paper shows that the number of sectors used to represent economic transactions influences the systemic metrics themselves.

Keywords Energy · Food - Economics · Information theory · Networks · Efficiency · Hierarchy · Equality · Redundancy $\cdot$ Biophysical $\cdot$ Complexity

\section{Introduction}

The consumption of energy, or more precisely power, the rate of consumption of energy, is a major factor in driving economic growth (Hall and Klitgaard 2012). Since the Industrial Revolution began in the UK in the late 1700s, humans have continued to annually consume more primary energy than the year before. Before the Industrial Revolution civilization was largely limited to energy technologies that harnessed solar-driven resource flows (sunlight, rivers, and wind) and stocks. Food and fodder, effectively storing sunlight via trees, plants, and crops, were the major fuels of the time for both human and animals to perform physical work. 
Many academic fields have a desire and role in understanding the role of energy, and energy-driven technology, in economic production and societal organization. Accordingly, this paper is motivated by the varying perspectives from these fields of inquiry.

\section{Econometric and Growth Modeling Perspective}

A multitude of econometric analyses collectively seem inconclusive as to the critical importance of energy in driving economic growth (Kalimeris et al. 2014). For example, approximately one-quarter of analyses fall into each of four possible causal domains between energy and gross domestic product (GDP): Growth in energy causes growth in GDP, growth in GDP causes growth in energy, no causality, and mutual causality. However, those studies that find a more pivotal and influential role for energy consumption in causing economic growth do so by including increasingly mechanistic and physically based views of how energy and technology work together.

Stern and Kander (2012) show that energy-driven capital plays the main role in describing Sweden's economic growth as it went through the industrial transition. Kümmel, Ayres, and Warr show that by including energy (or exergy) and its efficiency of conversion to useful (physical) work one creates significantly more accurate relationships linking energy, labor, and capital to economic output (Ayres and Warr 2005; Ayres 2008; Kümmel 2011). More recently, Santos et al. (2016) find that a further refinement considering useful exergy and labor as an input to capital (e.g., energy-driven machines) is a promising method for linking the role of energy to the economy that satisfies the need to describe economic output dependence upon energy while preserving mathematical requirements of economic aggregate production functions.

The implications of the work described in the previous paragraph are profound for modeling a transition to a lowcarbon energy system. Most macroeconomic models, such as those used to perform economic projections by federal governments and inform climate change mitigation, use a modeling framework that considers economic output as a function of capital, labor, and technological productivity (e.g., total factor productivity, TFP). This idea is usually derived from the so-called Solow growth model, or aggregate production function, developed in the 1950s (Solow 1956). However, most macroeconomic models assume TFP progresses into the future, at similar rates of the last several decades, while defining TFP only as all factors that are not otherwise described by capital and labor. The studies highlighted in the previous paragraph show that TFP is largely described by how efficiently energy is converted to useful energy services. Thus, for modeling the economy during an energy transition (e.g., to renewables and low-carbon energy) it is imperative that we no longer assume TFP is independent of feedbacks from the rate, cost, and efficiency implied by the energy system transition itself. I previously described how results of the Fifth Assessment of the Integrated Panel on Climate Change acknowledge yet neglect this important consideration [see Sect. 4.2 of King (2015a)].

In this paper, I take the perspective that energy, or power, is a requirement and can be a constraint, on economic growth because capital (e.g., machines in all sectors of the economy) requires energy carriers to operate. As such, if power constrains some economic possibilities, the structure of the economy might change with changes in power consumption.

\section{Net Energy Perspective}

Intuitively, energy carriers, or fuels, are needed to perform economic activity. Energy consumption is required to operate machines, drive cars and trucks, and refine metal ores into raw materials and final products. In fact, over the last fifty years, global real economic output (GDP) is approximated very closely as a linear function of primary energy consumption. The World Energy Council states that "As energy is the main fuel for social and economic development,..." (Council 2013). However, economic growth does not occur if energy is too expensive. Only two times since World War II has the world had energy expenditures greater than $8 \%$ of gross world product (late 1970s and 2008), and both were times of recession that spurred significant change and questions regarding economic growth (King et al. 2015b).

While many economists have used econometric methods to statistically relate energy consumption and technological factors to economic output, some ecologists and systems modelers take a different approach. This approach considers the intuitive notion that animals must obtain more energy in their food than they use in obtaining that food. That is to say that animals must have sufficient net energy from their food to survive when not feeding. If animals do not do this, then they die. The term "net energy" refers to the amount of energy left over after an energy production (e.g., gathering, refining, and distributing) process has consumed energy for its own operational purposes.

The net energy concept extends to describe the energy (and food) production systems within human-constructed industrial economies. Odum, Georgescu-Roegen, Hall, King and many others have applied this concept to describe energy production systems and technologies within the context of the overall economy (Odum 1971; GeorgescuRoegen 1971, 1979; Hall and Klitgaard 2012; Hall et al. 1986; King and Hall 2011; King 2010; King et al. 2015a, b; King 2015a). In particular, Hall has promoted the idea that "net energy" is an important metric that relates to societal structure and economic growth (Hall et al. 2009). 
Lambert et al. (2014) show that a net energy factor, combined with factors of gross power (GJ/capita/year) and inequality (Gini coefficient), seems to characterize average country-level health and welfare indices rather well. They also speculate on how much net energy is required from the energy system to support a given societal structure and attainment along a hierarchy of needs. The idea of relating gross and net energy to socioeconomic structure seems intuitive, yet it is poorly quantified for modern society. Hence, this lack of quantification is a motivation for this paper.

It is useful to take a historical perspective to understand the notion of net energy over the long term. Consider that the vast majority of preindustrial workers were part of the energy sector as agricultural labor. Thus, the size of energy sector relative to all non-energy sectors of preindustrial economies was much larger than today. Society was also highly unequal with a small number of landowners owning most of the wealth. Non-energy sectors and their workers are the consumers that purchase the net output (e.g., of food and fuels) from the energy and food sectors and workers. Preindustrial energy and food workers consumed much of their own production, and there was little to sell to others. Thus, there were relatively few 'others' in nonenergy sectors. Consequently, energy carriers were relatively expensive, and preindustrial economies spent large shares of total expenditures in producing food and energy. In preindustrial UK energy expenditures were equal to 35-45\% of economic output (King 2015a), and in Sweden in 1800 they were over $90 \%$ (Kander and Stern 2014). The net energy of the energy system had been low for several centuries, and thus, the structure of society had conformed its structure to that situation.

Coal-fired steam engines, introduced in the late 1700s, changed the net energy equation. They enabled coal to effectively power its own extraction by operating steampowered pumps to remove water from coal mines (Smil 2008; Fouquet 2008). This resource and technology combination began a 200+ year trend of fossil-fuel-driven machines powering a growing economy and population by enabling increasingly cheaper food and energy. Increasingly abundant and high net energy (i.e., abundant and affordable coal) energy enabled the technological changes that transformed the structure of economies from bases in agriculture to industry and services.

Today, the size of energy sectors relative to non-energy economic sectors is significantly smaller than 200 years ago, and this is largely because the energy system transformed from a low to a high net energy state. Consequently, post-World War II industrial economies usually spend less than an equivalent of $8 \%$ of gross domestic product (GDP) on primary energy (King et al. 2015b), and relative food expenditures declined until the around the turn of the millennium (King 2015b). It is this shift (rather, the tail end of it since 1947 in the USA) from a low to a high net energy system that I explore in this paper.

\section{Anthropological Perspective}

One anthropological perspective is provided by Joseph Tainter who has also posited the role of net energy in societal organization. He postulates that a society with an energy system (e.g., life cycle or supply chain) providing more energy relative to its own needs (e.g., higher net energy) has cheaper energy that enables more societal and/ or economic growth and complexity, ceteris paribus (Tainter 1988, 2011). Tainter (2011) states: "Our societies have changed from egalitarian relations, economic reciprocity, ad hoc leadership, and generalized roles to social and economic differentiation, specialization, inequality, and full-time leadership. These characteristics are the essence of complexity, and they increase the costliness of any society." Economists term this the division of labor. He further elaborates that "When human societies do have surplus energy, as industrial societies have over the past two centuries, it interacts with problem-solving to generate still more complexity. I term this the energycomplexity spiral," and "While there are many concepts of complexity in various sciences, the term is used here to mean (a) differentiation in structure (i.e., more parts to a system, and more types of parts); and (b) variation in organization, defined as constraints on potential ranges of behavior (Tainter 1988)."

Thus, the energy-complexity spiral of Joseph Tainter postulates that societies become more complex over time to solve problems, and more resources (e.g., energy) are required to create and maintain that complexity. Most of his insight comes from agrarian societies (e.g., Roman Empire), but this paper explores the possibility of measuring any structural characteristics of "complexity" within the modern economy (e.g., economic differentiation, specialization, and inequality).

\section{Economic Structure Perspective}

While Ulanowicz historically applied his methods to ecosystems and food chains, he recently ventured into the domain of economics in a recent paper considering monetary flows within sectors of Beijing, China (Huang and Ulanowicz 2014). The structure of input data and analysis of this paper is very similar to that of (Huang and Ulanowicz 2014). Also, McNerney and Fath (2013) have previously applied information theory and other network metrics to I-O matrices of multiple countries. However, McNerney and Fath (2013) were limited to either one or two years of $\mathrm{I}-\mathrm{O}$ tables for any given country. 
Another relevant analysis considers the "ubiquity" and "diversity" of imports and exports of countries to calculate a ranking of economic complexity (Hidalgo and Hausmann 2009). Hidalgo and Hausmann (2009) calculate metrics of economic complexity based only upon trade data by inferring that "...connections between countries and products signal the availability of capabilities in a country ..." The approach of the present paper is in some sense the exact opposite. I calculate structural metrics of a single economy by considering the internal structure of monetary flows (.e.g, I-O tables) rather than the import and export flows of the country along with those of all other countries to achieve a relative ranking. The advantage of my approach is that I only need data for a single country to assess that country. The disadvantage is that $\mathrm{I}-\mathrm{O}$ tables are less available for many countries than are the international trade data as used in Hidalgo and Hausmann (2009).

\section{Contribution and Organization of this Paper}

The contribution of this present paper is the analysis of the internal structure of a large economy over a long-term time series. It describes the structure of the U.S. economy as it relates to gross power input, economic output, and the expenditures of the energy and food sectors as economic proxies for net energy, or more specifically, net power. In performing this analysis, I investigate concepts of interest in many fields: ecological systems, economics, energy analysis, information theory, and anthropology. Further, a major contribution of this paper is to avoid speculating as to structural changes in society or an economy that might come with changes in net energy, but to measure them in a systematic manner and with minimal predetermined assumptions or modeling constructs.

This paper analyzes the flows among industries of the U.S. economy and quantifies the historical structural evolution for the largest economy in the world. To the knowledge of this author, this is only the second calculation of this type using information theory to characterize economic input-output tables [after Huang and Ulanowicz (2014)] and the first that characterizes a large economy over more than three decades. In addition, this paper contributes a new and more systematic quantification of "efficiency" and "redundancy" of network flows (see "Interpretive Quantifications of Distribution of I-O Table Entries" section) based upon the "phase space" described by the two major information theory calculations (see "Information Theory to Quantify Structure of Network Flows" section).

The first question pursued in this research was "Is there a changing structure of the U.S. economy from 1947 to 2012?" Upon answering this question affirmatively, I then asked more interesting questions. What are the trends and distribution patterns of that changing structure? Does the structure change in relation to gross or net power inputs and/or characterizations of the economy?

The U.S. economy structure is measured using the information theory framework of ecologist Ulanowicz, to be discussed further in "Information Theory to Quantify Structure of Network Flows" section. I apply Ulanowicz's calculations to the network of monetary flows among economic sectors of the U.S. input-output (I-O) tables as the base data that characterize the structure of the economy from 1947 to 2012. While the I-O tables do not measure all societal and cultural aspects that might relate to "complexity," they are one valuable characterization of structure as much as the flow of money among economic sectors is one way to describe our society.

The remainder of this article is organized as follows: "Methods" section describes the data and methods with subsections as follows: "I-O Data and Harmonization" section the process for harmonizing the I-O tables, "Energy and Net Energy" section a background on net energy and interpretation for this analysis, "Information Theory to Quantify Structure of Network Flows" section the information theory metrics, and "Interpretive Quantifications of Distribution of I-O Table Entries" section derivation of some additional metrics for interpreting the phase space of the information theory calculations. "Results" section describes results in several forms, and finally "Interpretation of Trends", "Implications of Modeled Number of Sectors" and "Organization and the Energy-Complexity Spiral" sections place the results into context of energy and the background material of this introducti on before "Concluding Remarks" section concludes the paper.

\section{Methods}

\section{I-O Data and Harmonization}

The core data for the analysis come from the U.S. inputoutput (I-O) tables of the Bureau of Economic Analysis (BEA). I use the summary tables rather than the full detailed tables. For the years 1947, 1958, 1963, and 1967, there is only a single I-O table (e.g., no Make and Use tables). For all other years, I base the calculations on the Use tables (rather than Make or Total Requirements tables). The harmonized $\mathrm{I}-\mathrm{O}$ tables are kept in nominal dollars. I focus on the structure of the $\mathrm{I}-\mathrm{O}$ tables by dividing all values by the total economic activity (or sum of all monetary flows in the tables). For these calculations, keeping the tables in nominal dollars poses no problems with inflation. I also perform calculations that scale with the total monetary flows in the $\mathrm{I}-\mathrm{O}$ tables. For those 
calculations (Eqs. 12, 17), I scale the results by a constant dollar index of chained real \$2009 using the GDP deflator from BEA Table 1.1.3 (BEA 2016).

Because the economy has changed over time (e.g., computers and information technology), the BEA has changed the definitions of the economic sectors. The Standard Industrial Classification (SIC) system was used to categorize economic sectors until 1992. Starting in 1997 the BEA categorized the benchmark I-O tables using the current North American Industry Classification System (NAICS), and I use the "before redefinitions" version of the NAICS Use tables.

Thus, an important step in this analysis was to create a set of harmonized I-O sectors that have a constant definition and number of sectors from 1947 to 2012 and contain as many sectors as possible. That is to say I wanted to keep as much richness from the $\mathrm{I}-\mathrm{O}$ summary tables as possible (given that they are already summarized from hundreds of subsectors into dozens of sectors).

The harmonized I-O tables were derived as a consistent 37-sector characterization of the U.S. economic I-O tables. I present one example of the harmonization process. The SIC-based summary tables (see Table S1) for 1947-1992 list sector numbers 5 (Iron and ferroalloy ores mining), 6 (nonferrous metal ores mining), 7 (coal mining), 9 (stone and clay mining and quarrying), and 10 (chemical and fertilizer mineral mining). I aggregate these into my sector number 4 within the 37-sector I-O framework. To match this sector number 4 using the NAICS-formatted I-O tables from 1997 to 2012 (see Table S2), I aggregated two of the NAICS summary table sectors: 4 (mining, except oil and gas) and 5 (support activities for mining). Here aggregation means that for the SIC tables and every column I added the entries for rows 5, 6, 7, 9, and 10 to create a single row. I performed the same for those numbered columns (for every row, I added the entries for those numbered columns). I performed the same process for the NAICS tables for the summary table rows 4 and 5 that are 'harmonized' into a new row number 4 within the 37 -sector framework. The mappings of summary sectors to each of the 37 harmonized sectors are listed in Supplementary Tables S1 and S2.

The aggregation of the summary table sectors into the 37-sector format was based on judgment as to the nature of the sectors. That is to say I performed no computational analysis, such as clustering (McNerney et al. 2013), to group the economic sectors. To explore the implications of the size of the network (e.g., the number of sectors) on the calculations in this paper, I also reduce the $\mathrm{I}-\mathrm{O}$ tables to 12 , 7, and 2 sector versions and report on the trends (see "Information Theory Trends as a function of Network Size" section for results and Tables S1 and S2 for sector descriptions).

\section{Modeling Inputs and Outputs (Exports and Imports)}

I calculate the information theory metrics (described in "Information Theory to Quantify Structure of Network Flows" section) for two boundaries of the I-O tables as network models. One considers the intermediate demands matrix only (e.g., only the $37 \times 37$ matrix of sectors). I refer to this as the "closed" or "intermediate" boundary because there is no concept of inputs to or outputs from this representation. The second "open" boundary uses value added and GDP per sector to model input into and output flows from the economy.

I use value added as input to each sector. The BEA defines value added as wages, taxes on production and imports less subsidies, and gross operating surplus (e.g., profits). I also include gross imports (as a positive input flow of money) as an input by removing that component from gross domestic product (GDP).

I use GDP as output from each sector. The BEA defines GDP as composed of personal consumption expenditures, private fixed investment, changes in inventories, government spending, and net exports (= exports-imports). Because I use gross imports as an input, I subtract them from GDP. I also move purchases from sectors "Federal general government" and "State and local general government" to output. In the I-O tables, these two government sectors have no sales, only purchases. The details of calculating sectoral inputs and outputs per year are listed in Supplementary Tables S3 and S4.

The information theory calculations are only defined for positive flows (e.g., of money) in networks (per Equations in "Information Theory to Quantify Structure of Network Flows" section). However, some I-O sectoral data (e.g., gross imports or exports) are presented as negative entries. In the case of each negative value for an input (e.g., gross imports), I convert it to a positive value and add it to an appropriate output (e.g., export) vector. Similarly, negative output entries (e.g., exports) are converted to positive inputs.

\section{Energy and Net Energy}

There are a multitude of studies discussing and analyzing the net energy of various energy technology and resource combinations. Usually, one or more energy return ratios (ERRs) or power return ratios (PRRs) are calculated for a given technology or system. The most common ERRs are energy return on (energy) invested (EROI) (Murphy et al. 2011), gross energy ratio (GER), net energy ratio (NER), and net external energy ratio (NEER) equal to EROI (King 2014; Brandt et al. 2013; King et al. 2015a). These ERRs characterize the total energy output divided by energy inputs over a life cycle or system under study. 
These energy inputs and outputs are the time integrals of the instantaneous power flows (often listed as annual power flows in data sets) during the life cycle. Thus, one can specify corresponding power return ratios (PRRs) as power output divided by power inputs (King et al. 2015a). In effect, the integral of a PRR over time achieves its respective ERR. For example, the integral of net power ratio (NPR) equals NER. For summaries of net energy analysis approaches and conceptual constructs, I direct the reader to the literature (Murphy et al. 2011; King 2014; Brandt et al. 2013; King et al. 2015a; Bullard and Herendeen 1975; Mulder and Hagens 2008; Modahl et al. 2013).

One goal of this analysis is to relate the changing structure of the U.S. economy over time to both net and gross energy consumption. More specifically, I concentrate on net and gross power (= energy/time) as the data in the I$\mathrm{O}$ tables are also per unit time (= dollars/year). Thus, I need to consider an economy-wide PRR. Individual resource (e.g., coal) or technology-specific (e.g., wind turbines) ERRs and PRRs will not suffice at this macro-system scale. Also, in order to holistically characterize the long-run role of energy in the economy it is necessary to simultaneously consider indicators of both food and what is usually meant by "modern" energy today.

I use an economy-wide PRR proxy in this paper that is the inverse of the energy and food sector expenditures divided by GDP (see Eq. 1) (King et al. 2015b). Fundamentally, ERRs are inversely related to cost, and PRRs are inversely related to price (King et al. 2015a). For example, if the U.S. food and energy sectors' spending is equivalent to $20 \%$ of GDP, then one can approximate the economywide NPR of the economy as $1 /(0.20)=5$. This metric is an economic proxy, hence subscript "economic," for a calculation using pure power units. For a pure energy system, the NPR is the net output of power (e.g., energy per year) divided by all intermediate system inputs needed to produce that power output (King et al. 2015a).

$$
\begin{aligned}
& \mathrm{NPR}_{\text {economic }}=\left(\frac{\text { Annual energy and food expenditures }}{\text { GDP }}\right)^{-1} \\
& =\frac{\text { GDP }}{\text { Annual energy and food expenditures }}
\end{aligned}
$$

For this paper, I calculate energy and food expenditures as the sum of purchases of five of the 37 sectors derived during the $\mathrm{I}-\mathrm{O}$ harmonization process: $1=$ Farms, $3=$ Oil and Gas extraction, $5=$ Utilities, $18=$ Food Products, Stores, and Services, and $23=$ Petroleum and Coal Products. Certainly some activities that are not energy and food are included in this aggregation (e.g., utilities includes water-related activity). However, the general trends are well characterized by these sectors.
For each year, there are two sums for energy and food expenditures, one for each of the closed and open-model formulations. Because the closed-model only considers the intermediate I-O matrix, the associated energy and food sector purchases are only those within the intermediate $\mathrm{I}-\mathrm{O}$ table. For the open-model, I additionally include expenditures from the inputs (as defined in "Modeling Inputs and Outputs (Exports and Imports)" section).

\section{Information Theory to Quantify Structure of Network Flows}

Over last few decades, Ulanowicz has established a body of work that uses information theory to describe the organization of ecological networks (Ulanowicz and Wolff 1991; Ulanowicz and Hannon 1987; Ulanowicz 2002; Ulanowicz et al. 2009; Ho and Ulanowicz 2005; Goerner et al. 2009). The value of this work is that it provides a method to understand the structure and organization of flows within networked systems from the standpoint of the trade-off between redundancy (via diversity of options) versus efficiency. This trade-off is measured by calculating aggregate system values based upon the network topology and flows between network nodes. In this paper, I use the information theory framework discussed much by Ulanowicz's to understand the organization of the U.S. economy as a network of monetary exchanges.

The main purpose of this "Information Theory to Quantify Structure of Network Flows" section is to ensure the reader is familiar with different terminologies that are used to refer to the same underlying mathematics of information theory. In "Information Theory Mathematics Background" section, I describe the formal descriptions from information theory (MacKay 2003) and relate these to the terminology and slightly different mathematics (for "conditional entropy" only) of Ulanowicz (2009) in "Closed Network Model Mathematics" and "Open Network Model Mathematics" sections. The mathematics are more important than the terminology, but it is important to understand terms used by different disciplines. Some readers might initially be more familiar with one set of terminology or another.

I refer the interested reader to the following works to become more fully familiar with the derivation, perspectives, and use of information theory for characterizing network structure. For terminology and derivation of the information theory metrics in "Information Theory Mathematics Background" section, see particularly Chapters 2 and 8 of (MacKay 2003). For Ulanowicz's definitions and use of the metrics (as applied to ecosystems) as in "Closed Network Model Mathematics" and "Open Network Model Mathematics" sections, see (Ulanowicz and Wolff 1991; 


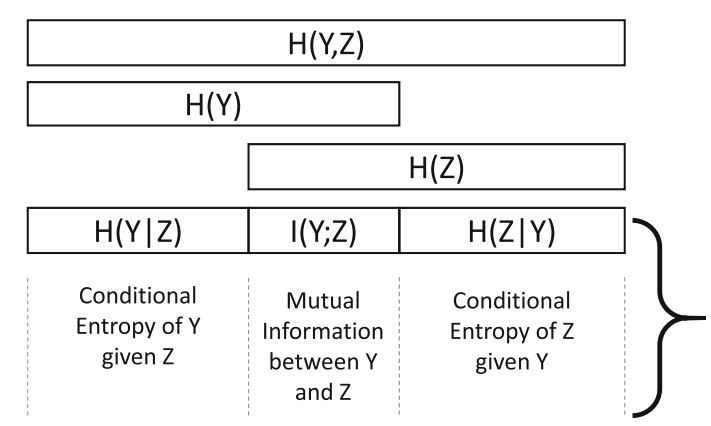

Fig. 1 Differences between the terminology of information theory (MacKay 2003) and the that of Ulanowicz (Ulanowicz et al. 2009) are made clear by showing the relationships among the quantities of joint

Ulanowicz 2004; Ulanowicz et al. 2009). Kharrazi's (2013) perspectives on information theory for network analysis of economic flows are also useful.

\section{Information Theory Mathematics Background}

The information theory concept is predicated on probability. The probability, $0 \leq p \leq 1$, that event $y$ will occur is $P(y) .{ }^{1}$ The (Shannon) information content in an outcome $y$ of a random variable is as in Eq. 2 and increases as the probability $P(y)$ approaches zero (MacKay 2003). ${ }^{2}$ MacKay (2003) (Table 2.9) explains information content via the example of the probability of letters, including a space, within an English document. Spaces and the letter ' $\mathrm{Z}$ ' occur with probabilities 0.1928 and 0.0007 , respectively, giving information content quantities from Eq. 2 of 1.65 and 7.26 nats, respectively.

$h(y)=\ln \left(\frac{1}{P(y)}\right)$.

Because there are multiple possible outcomes for any random variable (e.g., 26 letters of the alphabet as well as spaces and punctuation), the entropy, $H(Y)$, of the ensemble $Y$ is the average information content of all possible outcomes: ${ }^{3}$

\footnotetext{
${ }^{1}$ Here I use outcomes $y$ and $z$ as notation to reserve the letter $X$ to be consistent with nomenclature used by Ulanowicz.

2 In this paper, I use natural logarithms (giving units of 'nats') instead of logarithms of base 2 in MacKay (2003) (giving units of 'bits'). The difference in the calculated quantities and units for the information theory metrics is less important than the relative values of each metric when using equations with a consistent logarithm base. Thus, the difference between using logarithms of base 2 versus base $e$ has no relevance for the purposes of this paper.

${ }^{3}$ MacKay (2003) defines ensemble $X$ as a "...triple $\left(x, \mathcal{A}_{X}, \mathcal{P}_{X}\right)$, where the outcome $x$ is the value of a random variable, which takes on one of a set of possible values, $\mathcal{A}_{X}=\left\{a_{1}, a_{2}, \ldots, a_{i}, \ldots, a_{I}\right\}$, having
}

MacKay (2003)

Joint Entropy

(Marginal) Entropy of $Y$

(Marginal) Entropy of Z

Terminology of

MacKay (2003)
Ulanowicz et al. (2009)

Indeterminacy $=\mathrm{H}$

N/A

N/A

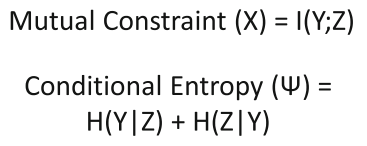

entropy (or indeterminacy), mutual information (mutual constraint), and conditional entropy. Figure concept taken from Figure 8.1 in MacKay (2003)

$H(Y)=\sum_{y \in \mathcal{A}_{Y}} P(y) \ln \left(\frac{1}{P(y)}\right)$.

Thus, the entropy of ensemble $Y, H(Y)$, is the weighted average of the information content of each outcome $y$ weighted by the probability of that outcome. MacKay (2003) notes that other names for the entropy of $H(Y)$ are the uncertainty or marginal entropy of $Y$. Importantly, it is a single metric of the entire ensemble, or set of probabilities. The quantity in Eq. 3 has also been referred to as information entropy and Shannon entropy.

For network analysis that considers the flow of some quantity (e.g., money, energy, materials, and information) from one node to another, one can define a single probabilistic event as a joint probability. The joint probability of a flow going from node $y$ to node $z$ could be expressed as $P(y, z)$. The single event is defined as two events, both $y$ and $z$, happening simultaneously. For an economic flow in an I-O table, the example is the probability of sector $z$ making a purchase from sector $y$.

The joint entropy of two ensembles $Y$ and $Z$ is:

$H(Y, Z)=\sum_{y z \in \mathcal{A}_{\mathcal{y}} \mathcal{A}_{\mathcal{Z}}} P(y, z) \ln \left(\frac{1}{P(y, z)}\right)$.

Ulanowicz refers to the mathematics of Eq. 4 as indeterminacy (Ulanowicz et al. 2009). ${ }^{4}$

Footnote 3 continued

probabilities $\mathcal{P}_{X}=\left\{p_{1}, p_{2}, \ldots, p_{i}, \ldots, p_{I}\right\}$, with $P\left(x=a_{i}\right)=p_{i}, p_{i} \geq 0$ and $\sum_{a_{i} \in \mathcal{A}_{X}} P\left(x=a_{i}\right)=1$." (MacKay 2003)

${ }^{4}$ Ulanowicz equation places a negative sign on the equation while moving $P(y, z)$ into the numerator within the logarithm, but it is mathematically equivalent to Eq. 4: $H(Y, Z)=\sum_{y z \in \mathcal{A}_{y} \mathcal{A}_{\mathcal{Z}}} P(y, z)$ $\ln \left(\frac{1}{P(y, z)}\right)=-\sum_{y z \in \mathcal{A}_{y} \mathcal{A}_{z}} P(y, z) \ln (P(y, z))$. 
MacKay (2003) Chapter 8 also defines conditional entropy of ensemble $Y$ given ensemble $Z, H(Y \mid Z),{ }^{5}$ as the average, over all $z$, of the conditional entropy of $Y$ given $z$. This quantity measures the average uncertainty that remains about $y$ when $z$ is known (see Eq. 5). One can also calculate the conditional entropy of $Z$ given $Y, H(Z \mid Y)$.

$H(Y \mid Z)=\sum_{y z \in \mathcal{A}_{\mathcal{Y}} \mathcal{A}_{\mathcal{Z}}} P(y, z) \ln \left(\frac{1}{P(y \mid z)}\right)$.

At this point, it is important to describe how MacKay (2003) and Ulanowicz use the same term of "conditional entropy" to actually refer to different mathematics. My representation of the very informative Figure 8.1 from MacKay (2003) demonstrates this distinction (see Fig. 1).

Ulanowicz's many papers refer to a term called conditional entropy of a network of flows that is in fact mathematically the same as adding the two conditional entropies when considering two ensembles. Equation (7) of Ulanowicz et al. (2009) specifies an equation for "conditional entropy" using symbol $\Psi$ (Eq. 9 in this paper), that is actually equal to the sum of both conditional entropies described above. Using Fig. 1, one can see that $\Psi=H(Y \mid Z)+H(Z \mid Y)$ (see Equation 9 for Ulanowicz's mathematics of $\Psi$ ).

With the terms defined in Eqs. 4 and 5 as well as Fig. 1, one can see the mathematical definition of what MacKay calls mutual information and Ulanowicz calls mutual constraint, $X$ [Equation (5) in Ulanowicz et al. (2009)]. These two terms refer to equivalent mathematics. From MacKay (2003) Chapter 8, for two ensembles, $Y$ and $Z$, the mutual information between $Y$ and $Z, I(Y ; Z)$, indicates the average reduction in uncertainty about $y$ that results from learning the value of $z$, or vice versa, the average amount of information that $z$ conveys about $y$ (MacKay 2003) (see Eq. 6). Mathematically, and demonstrated graphically in Fig. $1, I(Y ; Z)=I(Z ; Y)$.

$I(Y ; Z) \equiv H(Y)-H(Y \mid Z)$

\section{Closed Network Model Mathematics}

At this point, I shift to nomenclature used by Ulanowicz for those most familiar with that literature. The results of this paper are phrased using the equations discussed in this "Closed Network Model Mathematics" and "Open Network Model Mathematics" sections. Equations 7-12 describe the main calculations for information theory as used by Ulanowicz.

Take input-output matrix $T$ composed of entries $T_{i j}$ that are flows (of energy, material, money, etc.) from node $i$ (row $i$ ) to node $j$ (column $j$ ) in a network (Ulanowicz et al.

\footnotetext{
${ }^{5}$ For those unfamiliar, $H(Y \mid Z)$ is read as "the entropy of $Y$ given that $Z$ is known." The symbol $\mid$ is interpreted as "such that" or "given that" the following condition is already known.
}

2009). The dot subscript on $T$ indicates the sum of items over that dimension. For example, $T_{. j}$ is the sum of all flows into a given node $j$. Also, $T_{. .}$is the total system throughput (TST, of the flow type that is being modeled), or the sum of all flows in the network (see Eq. 10).

The system indeterminacy, $\mathrm{H}$ (Eq. 7), of the flows or "events," $T_{i j}$ is the same as the joint entropy. ${ }^{6}$ The system mutual constraint, $X$ (Eq. 8), measures the degree to which a system efficiently distributes flows among its nodes or its average degrees of constraint. ${ }^{7}$ The conditional entropy, $\Psi$ (Eq. 9), is a measure of the average degrees of freedom of a network for all flows $T_{i j}$, or the remaining choice of flow pathways for flows going from node $i$ to node $j$. Ulanowicz interprets $X$ as what is known about the network and $\Psi$ as what is not known, but what is possible in terms of flows moving through the network.

$$
\begin{aligned}
H & =-\sum_{i, j} \frac{T_{i j}}{T_{. .}} \ln \left(\frac{T_{i j}}{T_{. .}}\right) \\
X & =\sum_{i, j} \frac{T_{i j}}{T_{. .}} \ln \left(\frac{T_{i j} T_{. .}}{T_{i .} T_{. j}}\right) \\
\Psi & =-\sum_{i, j} \frac{T_{i j}}{T_{. .}} \ln \left(\frac{T_{i j}^{2}}{T_{i .} T_{. j}}\right) \\
T . . & =\sum_{i, j} T_{i j} \\
H & =X+\Psi
\end{aligned}
$$

Multiplying $H, X$, and $\Psi$ by $T$.. defines the system "capacity" $(C)$, "ascendency" $(A)$, and "overhead" or "potential reserve" $(\Phi)$ (see Eq. 12). The equation $\Phi=$ $\Psi T_{. .}$is often referred to more simply as "reserves." Mathematically capacity ( $H$ or $C$ ) for system development, or change, is the sum of the system's "constraints" ( $X$ or $A)$ plus "potential reserves" ( $\Psi$ or $\Phi)$.

$C=H T_{. .}=X T_{. .}+\Psi T_{. .}=A+\Phi$

Note that the maximum values for the information theory metrics for a closed N-node network system (with no inputs and outputs) are $H_{\max }=\Psi_{\max }=-\ln \left(1 / N^{2}\right)=$ $\ln \left(N^{2}\right)$ and $X_{\max }=\ln (N)$. There is no mathematical maximum for capacity, ascendency, and reserve because it is directly proportional to any inherent limit in total system throughput, TST.

\footnotetext{
${ }^{6}$ Indeterminacy of Eq. 7 is mathematically equivalent to joint entropy of Eq. 4.

${ }^{7} \mathrm{X}$, or mutual constraint, is mathematically equal to the terms mutual information and average mutual information (Shannon and Weaver 1962) that are used in the field of information theory.
} 


\section{Open Network Model Mathematics}

To include inputs and outputs to and from the network, respectively, the equations are modified as in Equations 13-18 to represent open networks (e.g., there are flows across the boundary that is defined by the flows among the nodes themselves) (Hirata and Ulanowicz 1984; Ulanowicz 2002):

$$
\begin{aligned}
O=- & \sum_{j} \frac{T_{\text {input } . j}}{T_{. .}} \ln \left(\frac{T_{\text {input } . j}}{T_{. j}}\right)-\sum_{i} \frac{T_{i, \text { export }}}{T_{. .}} \ln \left(\frac{T_{i, \text { export }}}{T_{i .}}\right) \\
& -\sum_{i} \frac{T_{i, \text { sink }}}{T_{. .}} \ln \left(\frac{T_{i, \text { sink }}}{T_{i .}}\right)
\end{aligned}
$$

$H_{\text {open }}=H+2 O$

$$
\begin{aligned}
& X_{\text {open }}=X+O \\
& \Psi_{\text {open }}=\Psi+O \\
& C_{\text {open }}=H_{\text {open }} \mathrm{TST}_{\text {open }}=X_{\text {open }} \mathrm{TST}_{\text {open }}+\Psi_{\text {open }} \mathrm{TST}_{\text {open }} \\
& =A_{\text {open }}+\Phi_{\text {open }}
\end{aligned}
$$

where

$$
\begin{aligned}
\mathrm{TST}_{\text {open }}= & T_{. ., \text {open }}=\sum_{i, j}\left(T_{i, j}+T_{i, \text { export }}+T_{i, \text { sink }}\right) \\
& =\sum_{i, j}\left(T_{i, j}+T_{\text {input }, j}\right) .
\end{aligned}
$$

Information Theory for Assessing Economic Flows

Much of the previous work by Ulanowicz (2009) originates from the ecological perspective that any given ecosystem must have a balance between efficiency and redundancy. In relatively more recent work, Ulanowicz et al. use different terminology to describe the terms in Eqs. 11 and 12 in the context of balanced economic systems (Goerner et al. 2009; Huang and Ulanowicz 2014). Goerner et al. (2009) use "systemic efficiency," SE, to describe ascendency, A, and "resilience capacity," RC, to describe potential reserves, $\Phi$. Also, Huang and Ulanowicz (2014), which investigates economic flows of Beijing, use the term "resilience" to label the term $\Phi=\Psi T_{\text {.. }}$.

Many times Ulanowicz and co-authors describe that there is a "window of vitality/viability" representing the range of balance within which ecosystems seem to structure themselves between too much efficiency versus resilience (Zorach and Ulanowicz 2003; Ulanowicz 2009; Goerner et al. 2009; Ulanowicz et al. 2009). If a system becomes too efficient, it is too brittle and unable to respond to change. If a system becomes too resilient or redundant, it has too much overhead in which to effectively grow and increase overall capacity for change.

Thus, it is natural to ask the question of balance for economies as ecosystems of monetary flows among firms or industries. Geoerner et al. (2009) discuss the information theory metrics in this economic context. They propose that total system "sustainability" (= capacity in Eq. 12) as the addition of "systemic efficiency" and "resilience capacity." Thus, they discuss both a size and structural component to assessing system sustainability, resilience (or overhead), and efficiency. The size component is total system throughput (Eq. 10). They use conditional entropy as the metric that characterizes the amount of structural (e.g., independent of size and normalized by TST) redundancy or overhead of a network. They use mutual constraint as the metric to characterize the amount of structural efficiency of a network.

Following Ulanowicz, my approach in this paper interprets the fraction of individual flow relative to the total system throughput as a probability. For example, considering a network with total flow (total system throughput, TST) of ten composed of ten individual flows of 1 , each is interpreted as having a probability of 0.1 . From the viewpoint of economic $\mathrm{I}-\mathrm{O}$ tables, the probability $P(y)$ of an event $y$ can be interpreted as the probability that a given economic sector will make a purchase form or sell its products to the other sectors. In addition, the joint probability of sector $z$ making a purchase from sector $y$ can be represented as $P(y, z)$, or using Ulanowicz's nomenclature the probability is $\frac{T_{i j}}{T}$ for a sector $j$ purchasing from sector $i$.

However, I have determined that there needs to be a more nuanced interpretation of how to use the information theory metrics to describe the balance between the efficiency and redundancy of network flows. The trade-off between efficiency and redundancy is not best-characterized by assuming mutual constraint, $X$, represents efficiency and conditional entropy, $\Psi$, represents resilience or redundancy. In addition, I describe another structural dimension with a trade-off between the hierarchy and equality of network nodes. These concepts and mathematics are now discussed in "Interpretive Quantifications of Distribution of I-O Table Entries" section.

\section{Interpretive Quantifications of Distribution of I-O Table Entries}

In this section, I describe what I term "interpretive metrics" based upon interpreting the phase spaced described by conditional entropy along one axis and mutual constraint as the second axis. These two metrics provide additional insight into the distribution of entries in the $\mathrm{I}-\mathrm{O}$ 


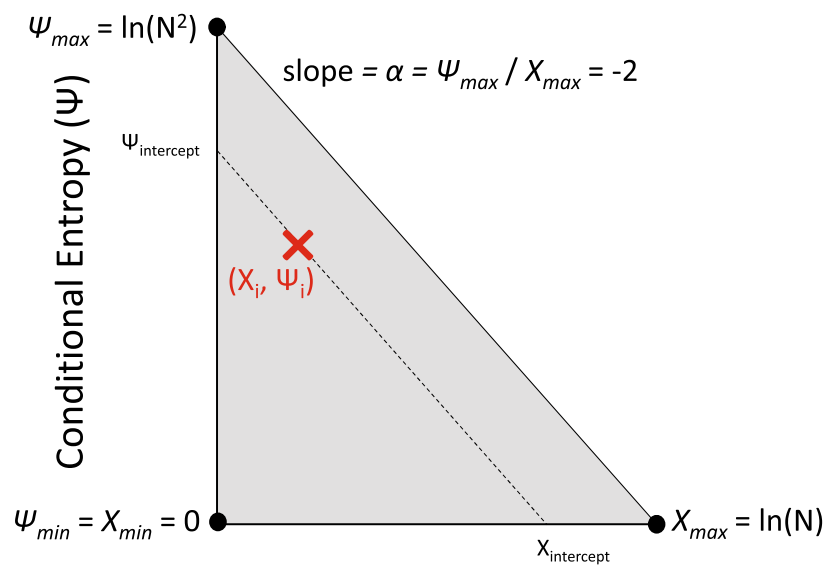

Mutual Constraint $(X)$

Fig. 2 For any set of flows in a closed network with $N$ nodes, the calculated information theory metrics reside in the lower left triangle phase space of a plot of conditional entropy versus mutual constraint

tables. They are interpretations of how to view the balance between mutual constraint and conditional entropy in the context of concepts such equality and hierarchy ("Equality and Hierarchy" section), and redundancy and efficiency ("Redundancy and Efficiency" section). The additional dimension of measurement beyond Ulanowicz's previous works is along a hierarchy-equality axis.

\section{The Phase Space for Information Theory Metrics}

For a given closed $N$-node network topology with each flow (or edge) of the same value (e.g., all flows are equal as represented by a uniform input-output matrix), then the network is at maximum conditional entropy $\left(\Psi_{\max }=\ln \left(N^{2}\right)\right)$, maximum indeterminacy $\left(H_{\max }=\ln \left(N^{2}\right)\right)$, and zero mutual constraint $\left(X_{\min }=0\right)$. This is the upper-left extreme point of Fig. 2.

For a network topology where each node is taking flow from one other node and giving that entire flow to another node, all flows of equal magnitude (e.g., a diagonal matrix is a mathematical example), then the network is at maximum mutual constraint $\left(X_{\max }=\ln (N)\right)$, zero conditional entropy $\left(\Psi_{\min }=0\right)$, and half of maximum indeterminacy $\left(H=\frac{1}{2} H_{\max }=\ln (N)\right)$. This is the lower right extreme point of Fig. 2.

For an I-O matrix with only a single flow (e.g., zeros in all flows except one), there is no longer a connected network, and both mutual constraint and conditional entropy are zero. This condition is the lower left point of Fig. 2. Thus, for any set of flows within a network, the information theory calculations reside in the shaded triangle phase space of Fig. 2.

For open network models, the endpoints of the upperright phase space boundary are not as simple to determine as in the case of the closed-model. The boundary is a function of the size of the network. For any given $N$, the boundary is defined by network flows that maximize $\Psi$ (for a uniform set of intermediate flows and equal inputs or outputs per node) or maximize $X$ (e.g., for a diagonal matrix of equal intermediate flows with equal inputs or outputs per node). This is the same as maximizing the distance of the upper-right boundary from the point $\left(X_{\min }, \Psi_{\min }\right)=(0,0)$. In this paper, I solved empirically for the equation for the upper boundary line for each size of network that I analyze (37-, 12-, 7-, and 2-sector I-O models). The equation is $\Psi=\Psi_{\text {openboundaryintercept }}-2 X$ where $\Psi_{\text {openboundaryintercept }}$ is approximately $7.884,5.934$, 5.046, and 3.138 for the 37-, 12-, 7-, and 2-sector openmodels, respectively.

\section{Equality and Hierarchy}

Close inspection of the patterns in network flows allows one to interpret a meaning for the upper boundary line of the phase space in Fig. 2. At both the points of $\Psi_{\max }$ and $X_{\max }$, as well as all points directly in between, each node of the network will meet two conditions with regard to throughput. First, the sum of input flows equals the sum of output flows. Second, all nodes have the same total input (and output) flow. Thus, each and every node has the same total throughput flowing through it. In this sense, each node is equal in total node flow throughput.

The interpretation of the upper-right phase space boundary line makes sense when it is considered a line of maximum indeterminacy (and conditional entropy) for a given mutual constraint. Thus, given just enough constraints to dictate a quantification of mutual constraint, maximum indeterminacy is achieved by distributing flows equally through all nodes as much as possible. At the point $\left(0, \Psi_{\max }\right)$, there are equal constraints on each flow, and each node has the same input from and output to each other node. At the point $\left(X_{\max }, 0\right)$, the constraint is that each node can have input flow from only one other node and provide output flow to only one other node. In between these two points are an infinite number configurations of network flows that meet the two conditions described in the previous paragraph.

Based upon this reasoning, I define an equation of equality as the proportion of the distance from the phase space point $\left(X_{\min }, \Psi_{\min }\right)=(0,0)$ (with equality of zero) to the maximum boundary line with equality equal to one. Lines of constant equality from zero to one have the same slope $(\alpha=-2)$ as the upper boundary line (see Fig. 2). Equation 19 provides a quantification of network node equality at a given a point $\left(X_{i}, \Psi_{i}\right)$ in the phase space. For 


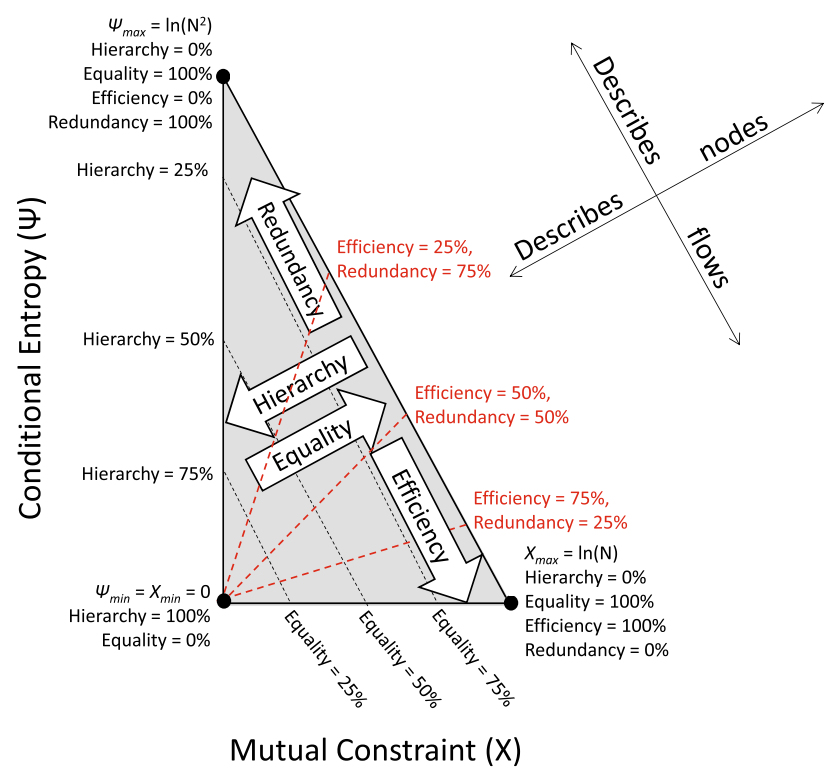

Fig. 3 Any movement in the closed network phase space along a line of constant node flow equality (and hierarchy) toward $\Psi_{\max }$ increases system flow redundancy while movement toward $X_{\max }$ increases system flow efficiency. Further, any movement in the phase space along a line of constant network flow efficiency (or redundancy) toward the upper-right boundary increases node flow equality while movement toward the origin of the lower left corner increases node flow hierarchy

open-models, $\Psi_{\max }$ and $X_{\max }$ are replaced by $\Psi_{\text {openboundaryintercept }}$, described previously, and $X_{\text {openboundaryintercept }}=\Psi_{\text {openboundaryintercept }} / 2$.

Equality $=\frac{X_{\text {Intercept }}}{X_{\max }}=\frac{\Psi_{\text {Intercept }}}{\Psi_{\max }}=\frac{\Psi_{i}+2 X_{i}}{\ln \left(N^{2}\right)}$

I define hierarchy as the opposite of equality, or one minus equality (see Eq. 20). In these definitions, hierarchy and equality are directly opposed. When one increases, the other must decrease. See Fig. 3 for a graphical representation of equality and hierarchy within the phase space.

$$
\begin{aligned}
\text { Hierarchy } & =1-\frac{X_{\text {Intercept }}}{X_{\max }} \\
& =1-\frac{\Psi_{\text {Intercept }}}{\Psi_{\max }} \\
& =1-\text { Equality } \\
& =1-\frac{\Psi_{i}+2 X_{i}}{\ln \left(N^{2}\right)}
\end{aligned}
$$

\section{Redundancy and Efficiency}

Here I define movement within the information theory phase space that characterizes the distribution of flows, rather than sum of all flows through each node. Constrained to any constant level of equality (e.g., line of slope -2 in Figs. 2, 3), changes in flows within the network will increase the redundancy of the network flows if they increase conditional entropy. Conversely, changes in network flows will increase the efficiency of the network flows if they increase mutual constraint. If not constrained to a given level of equality, then lines of constant efficiency (and redundancy) emanate from the origin of the phase space (see Fig. 3).

For a metric of efficiency, I use the "degree of order" (a) metric of Ulanowicz (see Eq. 21) (Ulanowicz 2009).

$$
\text { Efficiency }=a=\frac{X}{X+\Psi}=\frac{A}{A+\Phi}
$$

I define network flow redundancy as the opposite of flow efficiency, or one minus efficiency (see Eq. 22). In these definitions, redundancy and efficiency are directly opposed. When one increases, the other must decrease. Both efficiency and redundancy have values between and including zero and one.

$$
\begin{aligned}
\text { Redundancy } & =1-\text { Efficiency } \\
& =1-\frac{X}{X+\Psi} \\
& =\frac{\Psi}{X+\Psi}
\end{aligned}
$$

\section{Summary of Information Theory Metrics and Interpretation}

Here I present Table 1 as a summary of the information theory metrics presented in "Information Theory toQuantify Structure of Network Flows" and "Interpretive Quantifications of Distribution of I-O Table Entries" sections.

\section{Results}

In this section, I describe the results in several forms in order to provide a comprehensive viewpoint of the trends. First, "Information Theory Trends for the U.S. Economy" section describes the overall trends of the information theory metrics for the 37-sector harmonization of the U.S. Use input-output tables. Next, "Information Theory Trends as a function of Network Size" section discusses how the trends in the information theory metrics change with increasingly simplified representations of the economy as represented by decreasing the number of sectors used in more highly aggregated I-O tables. "3.4" section describes the results within the context of the allowable phase space described in Figs. 2 and 3. Finally, "Scaling Laws of U.S. Use Tables: Logarithmic Plots" section discusses the scaling laws of the 37-sector harmonized I-O tables, via logarithmic plots, and which economic sectors have the highest flows in the economy. 
Table 1 Summary of information theory metrics discussed in this paper: symbols, equations, terms, and descriptions of what the equations represent

\begin{tabular}{|c|c|c|c|}
\hline Symbol & Equation(s) & Term(s) & Description \\
\hline$h(y)$ & (2) & (Shannon) information content (MacKay 2003) & $\begin{array}{l}\text { An outcome } y \text { with lower probability has higher information } \\
\text { content }\end{array}$ \\
\hline$H(Y)$ & (3) & Entropy, uncertainty (MacKay 2003) & $\begin{array}{l}\text { The entropy of an ensemble is the average information content of } \\
\text { an outcome of that ensemble }\end{array}$ \\
\hline$H(Y, Z)$ & (4) & Joint entropy (MacKay 2003) & $\begin{array}{l}\text { The entropy of two ensembles, } Y \text { and } Z \text {, is the average information } \\
\text { content of two outcomes, } y \text { and } z \text {, occurring simultaneously }\end{array}$ \\
\hline$H$ & $\begin{array}{l}\text { (7) and } \\
\text { (14) }\end{array}$ & Indeterminacy (Ulanowicz et al. 2009) & $\begin{array}{l}\text { The entropy of two ensembles, } Y \text { and } Z \text {, is the average information } \\
\text { content of two outcomes, } y \text { and } z \text {, occurring simultaneously }\end{array}$ \\
\hline$H(Y \mid Z)$ & (5) & Conditional entropy (MacKay 2003) & $\begin{array}{l}\text { The average entropy (or uncertainty) about outcome } y \text { that remains } \\
\text { after outcome } z \text { is known }\end{array}$ \\
\hline$\Psi$ & $\begin{array}{l}\text { (9) and } \\
(16)\end{array}$ & Conditional entropy (Ulanowicz et al. 2009) & $\begin{array}{l}\text { The addition of average entropy about outcome } y \text { that remains after } \\
\text { outcome } z \text { is known to average entropy about outcome } z \text { that } \\
\text { remains after outcome } y \text { is known }\end{array}$ \\
\hline$I(Y ; Z)$ & (6) & Mutual information (MacKay 2003) & $\begin{array}{l}\text { The average reduction in uncertainty (or entropy) about } y \text { that } \\
\text { results from learning the value of } z \text {, or vice versa }\end{array}$ \\
\hline$X$ & $\begin{array}{l}\text { (8) and } \\
(15)\end{array}$ & Mutual constraint (Ulanowicz et al. 2009) & $\begin{array}{l}\text { The average reduction in uncertainty (or entropy) about } y \text { that } \\
\text { results from learning the value of } z \text {, or vice versa }\end{array}$ \\
\hline- & (19) & Equality & $\begin{array}{l}\text { (Describes nodes) Increases as the total system throughput is } \\
\text { distributed more evenly through each node }\end{array}$ \\
\hline- & $(20)$ & Hierarchy & $\begin{array}{l}\text { (Describes nodes) Increases as more of the total system throughput } \\
\text { of a network flows through fewer nodes }\end{array}$ \\
\hline- & $(21)$ & Efficiency & $\begin{array}{l}\text { (Describes flows) Increases as the flows in a network become more } \\
\text { concentrated to a small subset }\end{array}$ \\
\hline- & $(22)$ & Redundancy & $\begin{array}{l}\text { (Describes flows) Increases as the flows in a network become more } \\
\text { evenly dispersed }\end{array}$ \\
\hline$C$ & $\begin{array}{l}\text { (12) and } \\
\text { (17) }\end{array}$ & $\begin{array}{l}\text { Capacity (Ulanowicz et al. 2009), sustainability } \\
\text { (Goerner et al. 2009) }\end{array}$ & Indeterminacy, $H$, multiplied by total system throughput \\
\hline$A$ & $\begin{array}{l}\text { (12) and } \\
\text { (17) }\end{array}$ & $\begin{array}{l}\text { Ascendency (Ulanowicz et al. 2009), systemic } \\
\text { efficiency (Goerner et al. 2009) }\end{array}$ & Mutual constraint, $X$, multiplied by total system throughput \\
\hline$\Phi$ & $\begin{array}{l}\text { (12) and } \\
(17)\end{array}$ & $\begin{array}{l}\text { Overhead, reserve or potential reserve } \\
\text { (Ulanowicz et al. 2009), resilience capacity } \\
\text { (Goerner et al. 2009) }\end{array}$ & Conditional entropy, $\Psi$, multiplied by total system throughput \\
\hline
\end{tabular}

\section{Information Theory Trends for the U.S. Economy}

In this section, I report the time series of the information theory calculations and relate them to energy metrics for the U.S. economy. In general, the structure of the U.S. economy did in fact increase its structural reserves (conditional entropy, $\Psi$ ) and redundancy as it increased its gross power consumption and net power ratio $\left(\mathrm{NPR}_{\text {economic }}\right)$ until the end of the 1990s. Then, as NPR economic $_{\text {decreased, }}$ and gross power consumption stagnated, the economy expended (decreased) its reserves in order to increase its flow efficiency with increased mutual constraint and decreased conditional entropy.

Tables 2 and 3 summarize the trends, while Figs. 4 and 5 plot the information theory ("Information Theory to Quantify Structure of Network Flows" section) and interpretive metrics ("Interpretive Quantifications of
Distribution of I-O Table Entries" section), respectively, versus time, gross power consumption, and NPR economic of the U.S. economy.

I perform two time series calculations, and the figures and tables display results for both. The first time series calculation considers only the intermediate I-O transactions without any defined inputs or outputs ("closed: intermediate only"). The second time series calculation ("open: including value added and GDP") includes the sectoral value added components plus gross imports of the I-O tables as network inputs along with the sectoral GDP minus imports of the $\mathrm{I}-\mathrm{O}$ tables as network outputs. This open-model mimics that of Costanza and Herendeen in examining the embodied energy of the U.S. economic sectors as one expands the boundary to include more aspects of the I-O tables (Costanza 1980; Costanza and Herendeen 1984). 
Table 2 Information theory metrics of the 37-sector harmonized U.S.

\begin{tabular}{lllll}
\hline Phase & Years & Indeterminacy $(\mathrm{H})(\% /$ year $)$ & Mutual constraint $(\mathrm{X})(\% /$ year $)$ & Conditional entropy $(\Psi)(\% / y e a r)$ \\
\hline \multicolumn{2}{l}{ Closed: intermediate transactions only } & & +0.57 \\
1 & $1947-1967$ & +0.38 & -0.32 & +0.09 \\
2 & $1967-2002$ & -0.07 & -0.79 & -0.60 \\
3 & $2002-2012$ & -0.35 & +1.2 & +0.20 \\
Open: with inputs (value added & + imports) and outputs (GDP-imports) & -0.18 \\
1 & $1947-1967$ & +0.08 & -0.36 & -0.44 \\
2 & $1967-2002$ & -0.31 & -0.86 & +0.61 \\
3
\end{tabular}

Use I-O tables (H, indeterminacy; $\mathrm{X}$, mutual constraint; $\Psi$, conditional entropy). The rates listed are linear rates of change over the time period indicated (e.g., $\left(H_{1967}-H_{1947}\right) / 20$ years)

Table 3 Interpretive information theory metrics of the 37-sector harmonized U.S

\begin{tabular}{|c|c|c|c|c|c|c|c|}
\hline Phase & Years & $\begin{array}{l}\text { Gross power } \\
\text { (\%/year) }\end{array}$ & $\begin{array}{l}\text { Net power ratio } \\
\text { (\%/year) }\end{array}$ & $\begin{array}{l}\text { Hierarchy } \\
\text { (\%/year) }\end{array}$ & $\begin{array}{l}\text { Equality } \\
\text { (\%/year) }\end{array}$ & $\begin{array}{l}\text { Redundancy } \\
\text { (\%/year) }\end{array}$ & $\begin{array}{l}\text { Efficiency } \\
\text { (\%/year) }\end{array}$ \\
\hline \multicolumn{8}{|c|}{ Closed: intermediate transactions only } \\
\hline 1 & $1947-1967$ & +4.1 & +2.8 & -1.8 & +0.26 & +0.17 & -0.65 \\
\hline 2 & $1967-2002$ & +1.9 & +3.4 & +2.1 & -0.18 & +0.17 & -0.74 \\
\hline 3 & 2002-2012 & -0.3 & -1.8 & +1.0 & -0.16 & -0.26 & +1.6 \\
\hline \multicolumn{8}{|c|}{ Open: with inputs (value added + imports) and outputs (GDP - imports) } \\
\hline 1 & 1947-1967 & +4.1 & +2.5 & -0.01 & 0.00 & +0.12 & -0.44 \\
\hline 2 & $1967-2002$ & +1.9 & +3.2 & +0.68 & -0.40 & +0.15 & -0.61 \\
\hline 3 & 2002-2012 & -0.3 & -1.0 & +0.19 & -0.16 & -0.17 & +0.92 \\
\hline
\end{tabular}

Use I-O tables (hierarchy, equality, redundancy, and efficiency). The rates listed are linear rates of change over the time period indicated (e.g., $\left(x_{1967}-x_{1947}\right) / 20$ years)
Each table summarizes trends for the information theory metrics by three temporal phases (discussed in the following section) that emerge from the results. Figure 6 plots the numbers from Tables 2 and 3 to visualize how the rates of change in the metrics fall into the three phases of structural change (for the 37-sector model). One can see a general counter clockwise trend along the progression of the phases in Fig. 6.

\section{Closed Network Results (Intermediate Transactions Only)}

There are interesting relationships between U.S. domestic economy information theory metrics as compared to both gross power consumption and the economy-wide measure of net power ratio. In interpreting the results of Table 2, recall from "Information Theory to Quantify Structure of Network Flows" section that the maximum values for a 37node network are $\mathrm{H}_{\max }=\Psi_{\max }=\ln \left(N^{2}\right)=\ln \left(37^{2}\right)=$ 7.22 and $X_{\max }=\ln (37)=3.61$.

There are three major phases that can describe the economic structure of the U.S. economy as they relate to net and gross power consumption (see Fig. 6). The three phases are defined by unique combinations of directional change for the interpretive information theory metrics. During Phases 1, 2, and 3, the change in node flow hierarchy was,-+ , and + , respectively (and opposite for equality), while the change in redundancy was,++ , and - , respectively (and opposite for efficiency). The same pattern of 3 distinct sign combinations almost emerges when considering mutual constraint $(-,-,+)$ and conditional entropy $(+,+,-)$ where the inconsistent change is a low positive change for conditional entropy for Phase 2 (instead of a negative change).

In Phase 1 (1947-1967) from the end of World War II until the end of the 1960s, both gross power consumption and the economic net power ratio (NPR economic) of the economy were growing at relatively high rates of 4.1 and $2.8 \% / y e a r$, respectively (e.g., the intermediate cost share of energy and food was declining relatively rapidly). Indeterminacy $(\mathrm{H})$ and conditional entropy $(\Psi)$ were increasing at relatively high rates, while mutual constraint (X) was declining at what can be viewed as a moderate rate compared to subsequent years. At the same time, node 


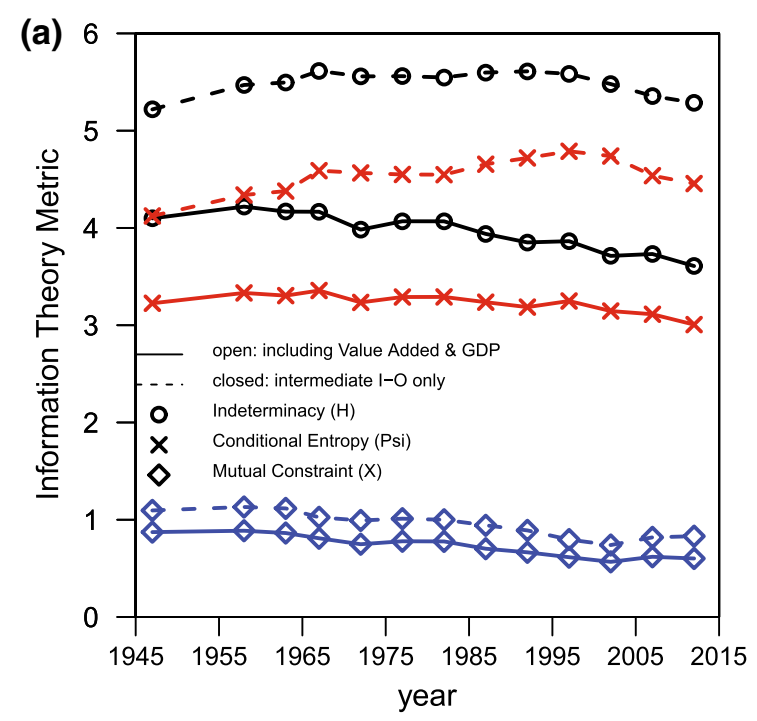

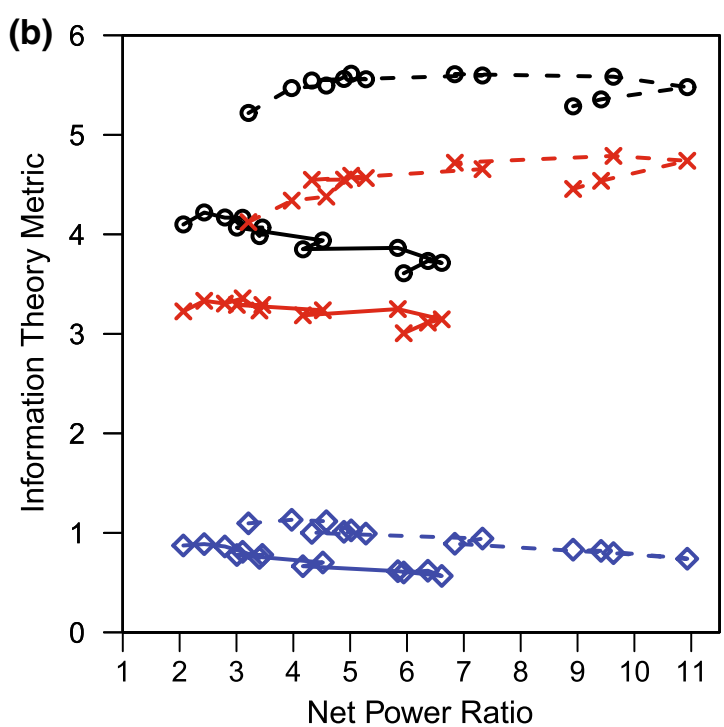

Fig. 4 A plot of the harmonized 37-sector U.S. Use I-O matrix information theory metrics versus both gross power consumption and an economy-wide metric of net energy shows three distinct phases. Solid lines show "open" boundary results that include per-sector

hierarchy was declining (equality was increasing) at a relatively high rate, while flow efficiency was decreasing (redundancy was increasing) (see Table 3).

Phase $2(1967-2002)$ spans the time from the end of the 1960s through the end of the second Millennium. It marks a separate phase of U.S. economic structure in that the rate of change in one indicator has changed signs. Hierarchy is now increasing (and equality is decreasing), the opposite of Phase 1. Indeterminacy and conditional entropy have changed from high to low rates of increase, and mutual constraint has started declining more rapidly than before. Efficiency and redundancy are changing similarly as in Phase 1.

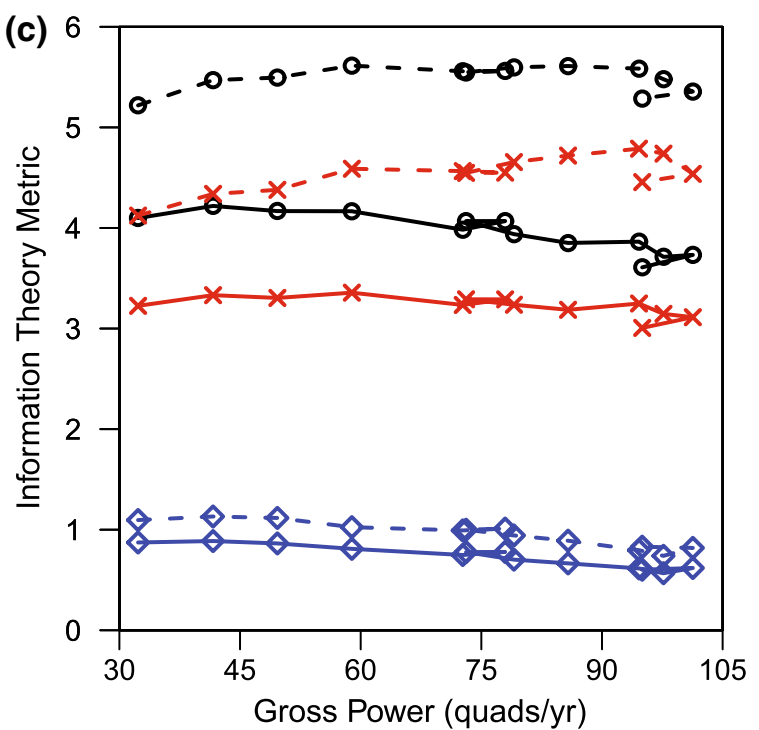

value added plus imports as inputs into each sector and per-sector GDP minus imports as outputs from each sector. Dashed lines show "closed" boundary results that consider the intermediate I-O transactions only

From an energy perspective and compared to Phase 1, gross power consumption and net power ratio continued to increase in Phase 2 but at half the rate for gross power and a slightly higher rate for net power ratio.

Phase 3 (2002-2012) represents the (short) trend since the beginning of the twenty-first century. This time period is characterized by the change from an increasing to a decreasing trend for both gross power consumption $(-0.3 \% / y e a r)$ and net power ratio $(-1.8 \% / y e a r)$. Interestingly, this change in direction of the rates of change in the power metrics coincides with significant changes in trends for the information theory metrics. Indeterminacy 


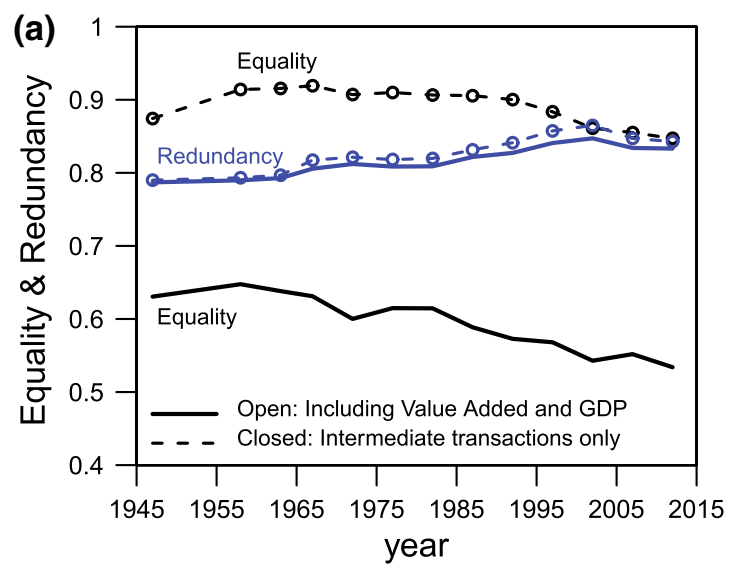

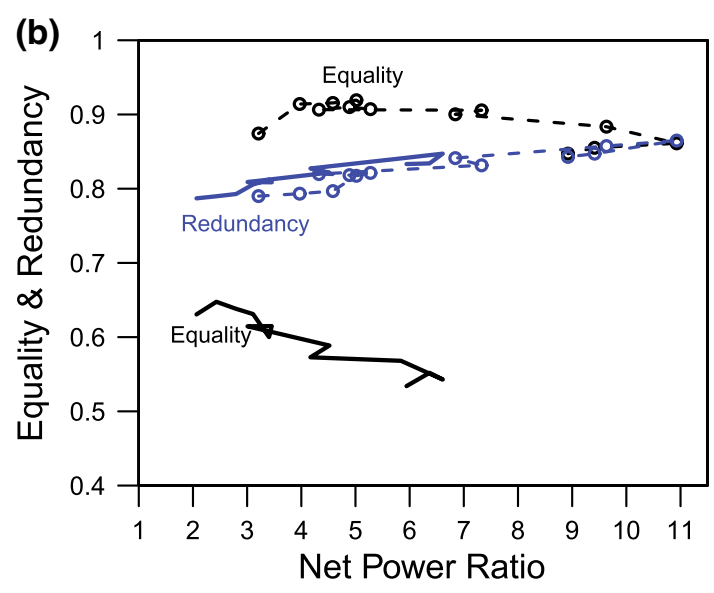

Fig. 5 A plot of the harmonized 37-sector U.S. Use I-O matrix interpretive information theory metrics versus both gross power consumption and an economy-wide metric of net energy shows three distinct phases. Solid lines show "open" boundary results that include

(a)

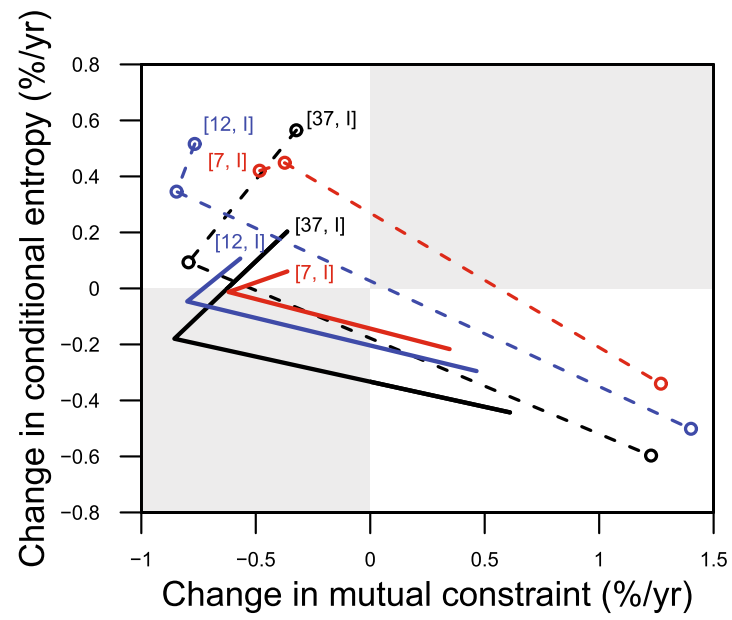

Fig. 6 The three phases are illustrated by plotting the rates of change in a conditional entropy versus mutual constraint and $\mathbf{b}$ redundancy versus equality. The 37 -sector rates of change for both figures progress in a counterclockwise manner between quadrants, and it is this

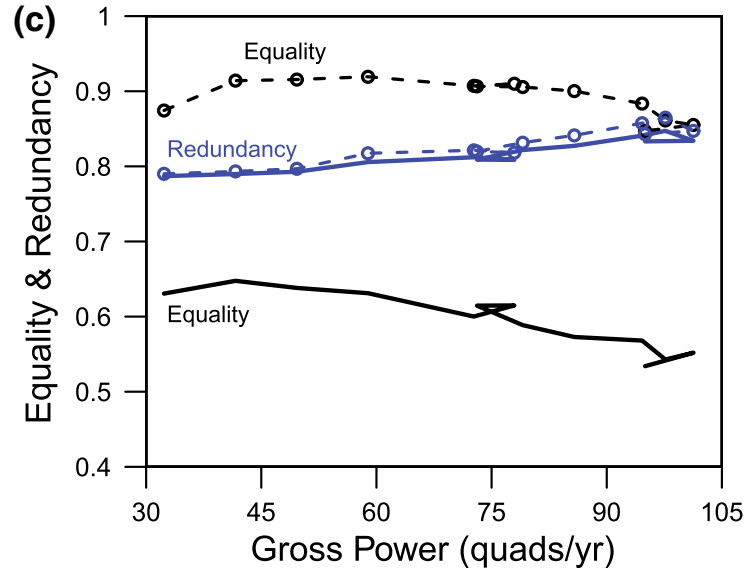

per-sector value added plus imports as inputs into each sector and persector GDP minus imports as outputs from each sector. Dashed lines show "closed" boundary results that consider the intermediate I-O transactions only

(b)

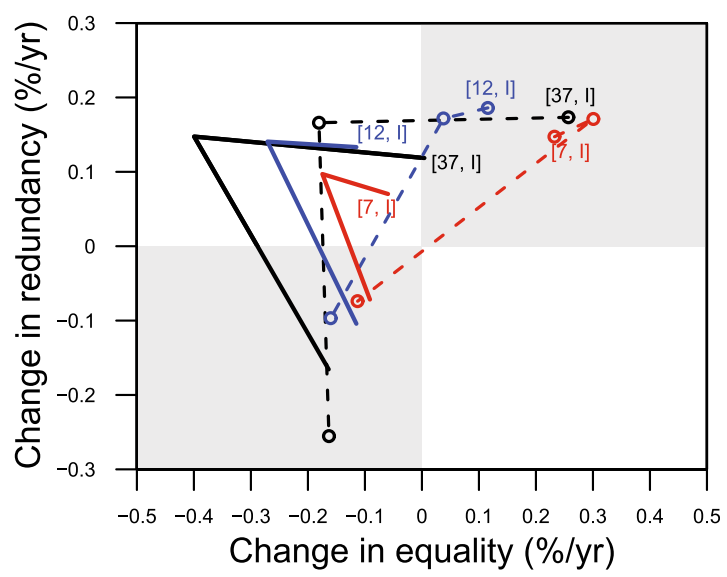

positioning of the phases in different quadrants that indicates the phases. All Phase 1 points are labeled as "[37,I]" meaning the Phase 1 rates of change for the 37 -sector aggregation 
begins to decline as rapidly as it increased in Phase 1 , and the rates of change in mutual constraint and conditional entropy change sign. In addition, the change in redundancy is now negative (change in efficiency is positive). Hierarchy and equality have rates of change similar to those in Phase 2.

\section{Open Network Results (Including Value Added and GDP Per Sector)}

The trends of the rates of change in the open information theory metrics generally follow those of the closed network model (see Tables 2, 3; Figs. 4, 5, 6). The net power ratio now includes expenditures for value added and gross imports and thus is a different value but with similar rates of change over time as in the closed-model. The trends in hierarchy, equality, redundancy, and efficiency are all very similar between the open- and closed-models, but with some differences.

One difference concerns conditional and indeterminacy. Indeterminacy generally decreases over time for the openmodel from 1958, but it does not clearly follow a decreasing trend for the closed-model until 1992. The trends of indeterminacy per phase are similar between the open- and closed-model except for a faster rate of decline in Phase 2 for the open-model. This difference for Phase 2 is driven by conditional entropy that declines in Phase 2 for the open-model, but slightly increases for the closedmodel.

(a)

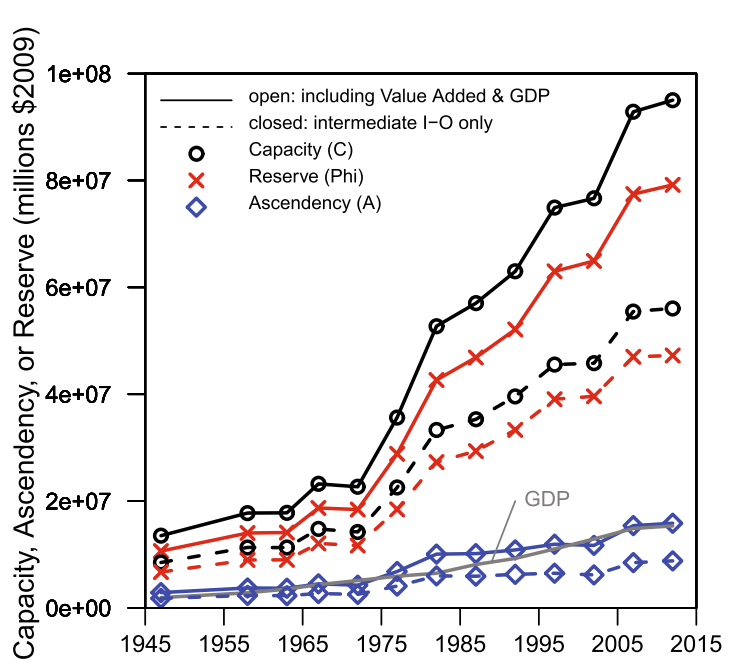

The other difference of note is that open-model hierarchy and equality are approximately the same at the end of Phase 1 as at the beginning, whereas there is a clear increasing trend for equality (decreasing for hierarchy) in the closed-model.

\section{Information Theory Trends Scaled by Total System Throughput}

This section summarizes the results for the information theory metrics described by the previous section after each is multiplied by the total system throughput $\left(\mathrm{TST}=T_{\text {.. }}\right.$ ). The reason to consider this perspective is to understand whether changes in network structure occur simultaneously with absolute growth. Even if the structure of network flows themselves changes, perhaps they do this to preserve, or at least occur simultaneously with, absolute growth.

Figure 7 shows ascendency (A), potential reserve $(\Phi$, also referred to as overhead), and capacity (C) over time for the 37-sector harmonized I-O tables. Table 4 indicates the rates of change in the metrics, per temporal phase, as well as the rate of change in GDP.

Even as the economy's structure changed per the three phases discussed, there is only one instance in which all three scaled metrics do not increase from one benchmark year to the next: from 1967 to 1972 (see Fig. 7 and data of Supplemental Information). Thus, both the scaled and unscaled information theory metrics indicate 1967 and/or 1972 as a critical transition point, and I believe using 1967 as a breakpoint shows more distinct mathematical differences. (b)

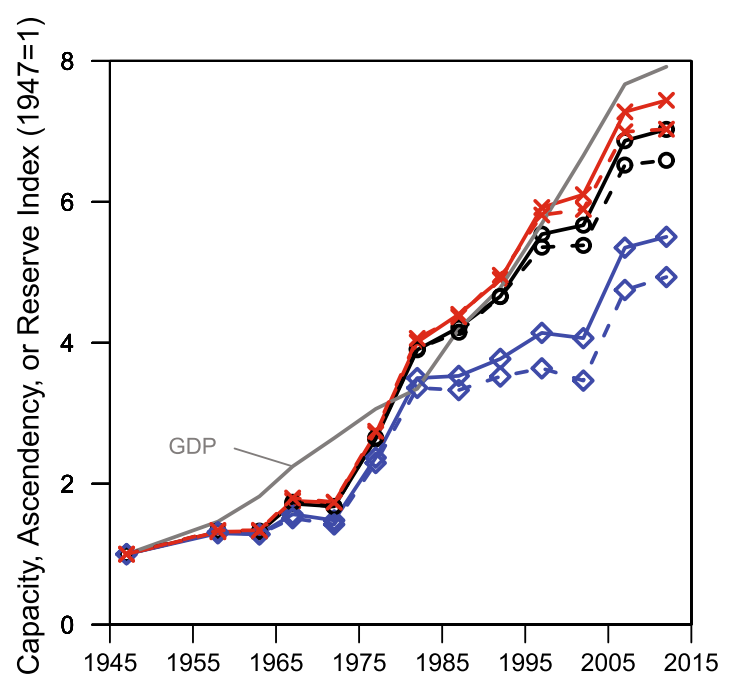

Fig. 7 A plot of information theory metrics as multiplied by total system throughput for the 37-sector harmonized I-O tables: a Units of constant $\$ 2009$, b indexed to 1947. The dashed lines with circles represent the closed-model results, and solid lines represent the open-model results 
Table 4 Information theory metrics, multiplied by TST, of the 37-sector harmonized U.S.

\begin{tabular}{|c|c|c|c|c|c|}
\hline Phase & Years & GDP (\%/year) & Capacity (C) (\%/year) & Ascendency (A) (\%/year) & Potential reserve $(\Phi)$ (\%/year) \\
\hline \multicolumn{6}{|c|}{ Closed: intermediate transactions only } \\
\hline 1 & $1947-1967$ & +6.2 & +3.7 & +2.6 & +4.0 \\
\hline 2 & $1967-2002$ & +5.6 & +6.0 & +3.7 & +6.5 \\
\hline 3 & 2002-2012 & +1.9 & +2.2 & +4.2 & +1.9 \\
\hline \multicolumn{6}{|c|}{ Open: with inputs (value added + imports) and outputs (GDP-imports) } \\
\hline 1 & $1947-1967$ & +6.2 & +3.6 & +2.8 & +3.8 \\
\hline 2 & 1967-2002 & +5.6 & +6.6 & +4.6 & +7.0 \\
\hline 3 & 2002-2012 & +1.9 & +2.4 & +3.5 & +2.2 \\
\hline
\end{tabular}

Use I-O tables (C, capacity; A, ascendency; $\Phi$, (potential) reserve). The rates listed are linear rates of change over the time period indicated (e.g., $\left(x_{1967}-x_{1947}\right) / 20$ years $)$

Ascendency slightly decreases (for both open- and closedmodels) from 1958 to 1963 as well as from 1997 to 2002 . For the closed-model, only ascendency decreases from 1982 to 1987 and capacity slightly decreases from 1958 to 1963.

There are a few trends of note for these metrics that provide additional insight over those not scaled by TST in "Information Theory Trends for the U.S. Economy" section. First, GDP follows ascendency in magnitude (Fig. 7a). This is not surprising as ascendency captures the known structure of a network (e.g., the economy), and GDP is usually roughly equivalent to the sum of all intermediate transactions.

Second, GDP is nearly equal to the closed ascendency in 1947 , but by 1997 it is closer to open-model ascendency. There are some fluctuations in between, but the overall trends seem to be for open-model ascendency to now be nearly equivalent to GDP while closed-model ascendency is almost half of GDP.

Third, GDP outpaced all information theory metrics through 1972 (roughly Phase 1, see Table 4; Fig. 7b) before increasing at a similar high rate as capacity and reserve during Phase 2 and similar lower rate in Phase 3. Ascendency increased more slowly than GDP in Phase 2, but much more quickly in Phase 3. The 1977 and 1982 benchmark years produce somewhat outlying rates of increase for all three metrics, and a full explanation requires further research.

Finally, the reserve metric changed at a higher rate than both capacity and ascendency, and it most closely matches the rate of change in GDP (see Fig. 7b). If we consider this metric more specifically as "potential reserve" in that all of it is not useful, then perhaps it indicates the U.S. economy still has some significant structural reserve of which to make use, even after the structural changes since 2002 .

\section{Information Theory Trends as a function of Network Size}

To explore the ramifications of the size of the network on the information theory metrics, I show results for additional harmonizations of the U.S. Use tables that are aggregated into fewer total sectors. The purpose is to understand the impacts of increasingly simplified representations of the economy. This comparison is valuable because economists often simplify models of economies in order to explore certain trends and hypotheses. Thus, one can ask whether simplified models of economies, defined here as having fewer sectors, might miss or differently describe important systemic characteristics. The detail of the model (or data) might influence the interpretation of system metrics.

As one increases the size of the network, one reaches diminishing returns on the maximum values attainable for the information theory metrics. Since maximum indeterminacy (for the closed-model) $H_{\max }=-\ln \left(1 / N^{2}\right)=\ln \left(N^{2}\right)$, as $N$ becomes large, $H_{\max }$ increases more slowly. For example, in moving from a 2- to 3-node representation, $H_{\max }$ increases by 0.8 , but $H_{\max }$ increases by only 0.05 when moving from a 36- to 37-node representation.

Figures 6, 8, and 9 compare the information theory metrics for the 37-, 12-, and 7-sector models (see Supplemental Figures S1-S2 for the two sector plots). The openmodel 12- and 7-sector plots generally follow the same trends as the 37 -sector open harmonization, providing confidence that the aggregations capture the structure of the I-O tables relatively consistently over time. However, the 12- and 7-sector closed-model harmonizations show some distinctly different trends from the 37-sector model.

Figure $8 \mathrm{c}$ shows perhaps the most powerful link between net energy and economic structure. Mutual 
(a)

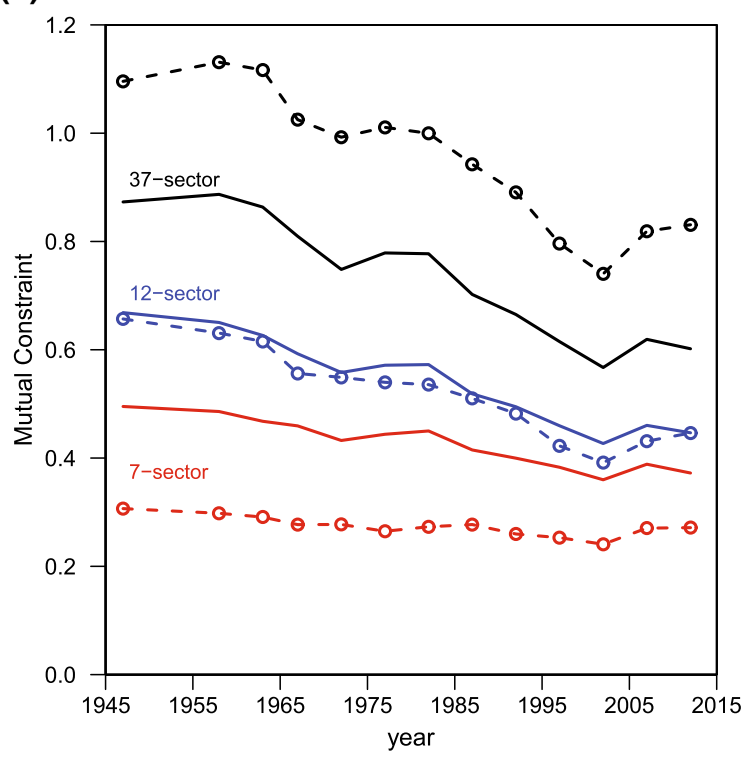

(b)

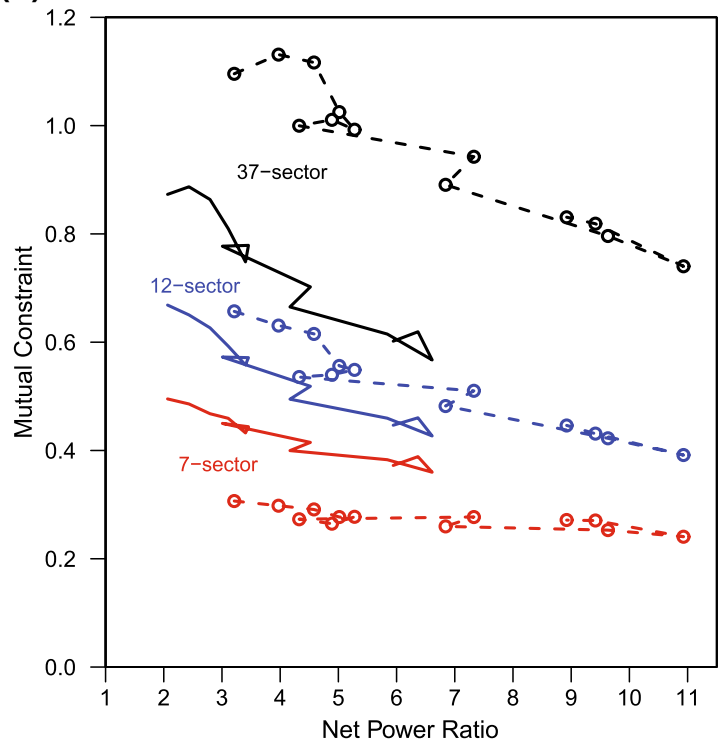

Fig. 8 A plot of the a mutual constraint and $\mathbf{b}$ conditional entropy versus time, as well as both versus net power ratio, c, d, respectively, for the 37-, 12-, and 7-sector representations of the U.S. Use I-O

constraint seems to be almost a linear function (with negative slope) of $\mathrm{NPR}_{\text {economic }}$ for all aggregation levels. There was a period of stagnation from 1967 to 1982 when $\mathrm{NPR}_{\text {economic }}$ did not change much, and a clear increase after 2002 when NPR $_{\text {economic }}$ had reached its maximum.

Tables 5, 6, 7, 8, 9, 10, 11, 12, 13, and 14 show correlations between the various representations of the U.S. Use tables. If pairs of time series for each harmonized representation of the U.S. Use tables have high correlation (b)

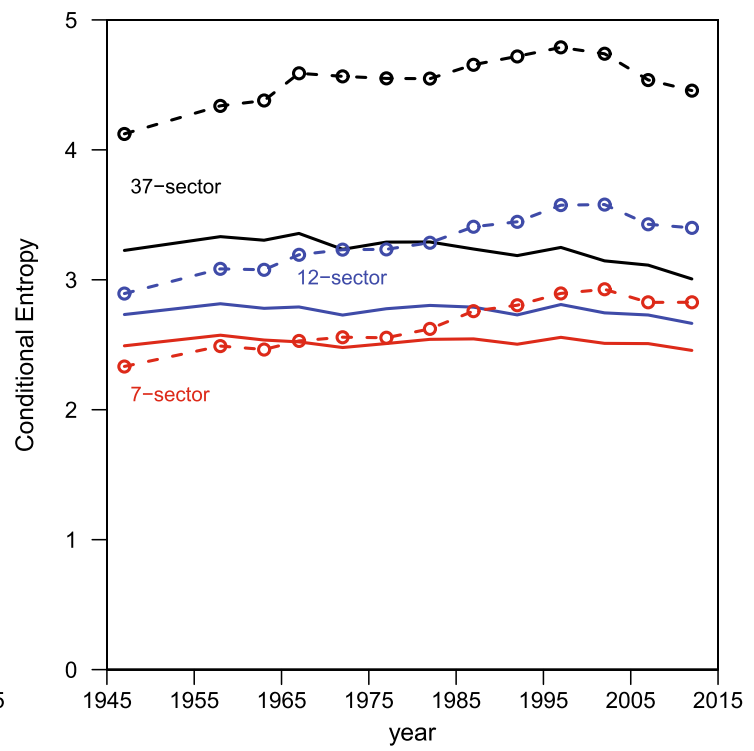

(d)

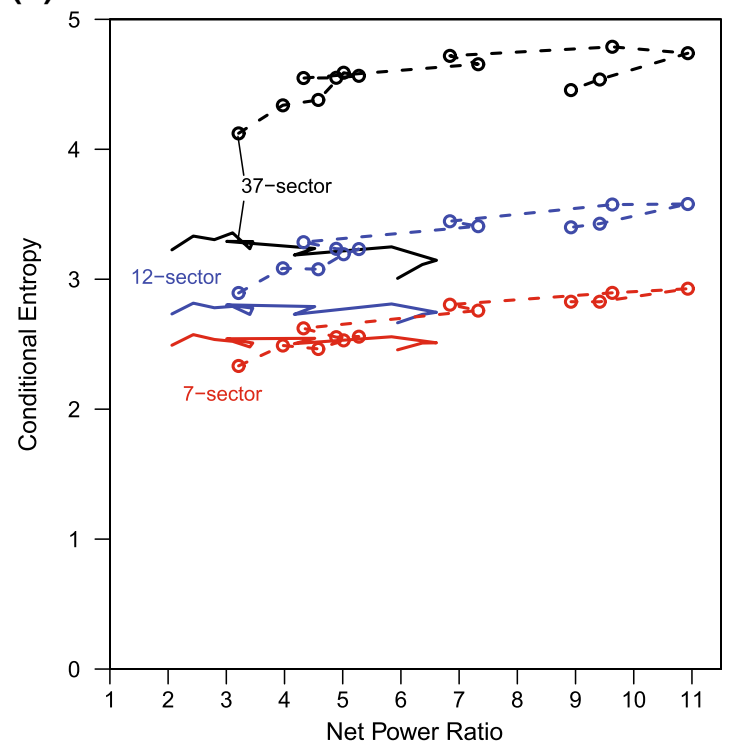

tables. The dashed lines with circles represent the closed-model results, and solid lines represent the open-model results

(either positive or negative), then they similarly characterize changes in the economy. I discuss these tables in more detail in the following subsections.

\section{Open-Model Results (Including Value Added and GDP Per Sector)}

For the open-model results, one of the first takeaways is that the correlations are generally high for the 37-, 12-, 


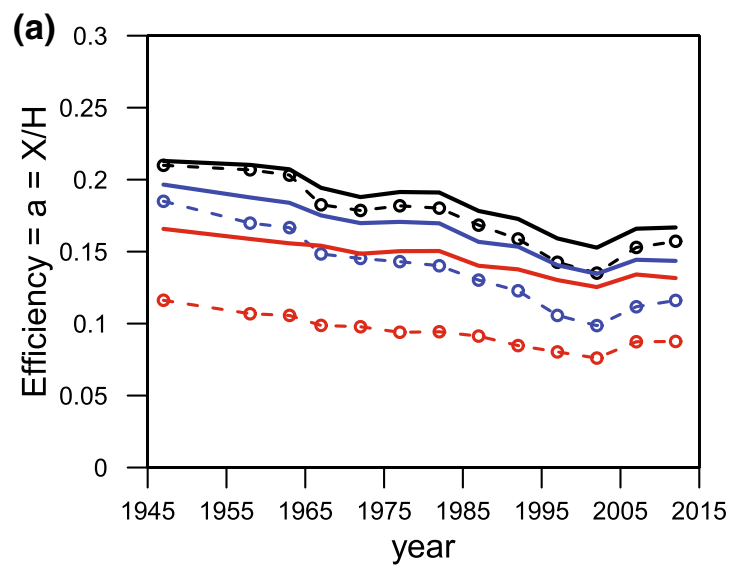

Fig. 9 A plot of calculations for the 37-, 12-, and 7-sector representations of the U.S. Use I-O tables for a efficiency (37-, 12-, and 7-sector results run from top to bottom) and $\mathbf{b}$ equality over

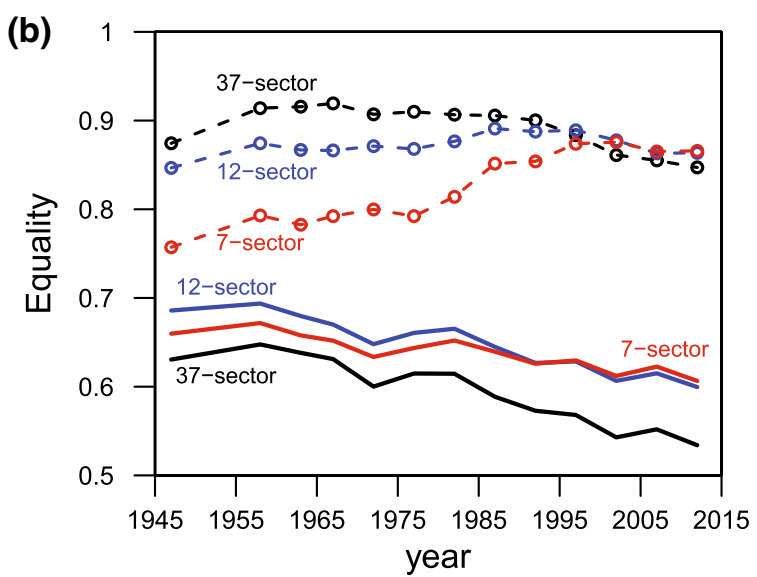

time. The dashed lines with circles represent the closed-model results, and solid lines represent the open-model results

Table 5 Table showing correlations for 37-, 12-, 7-, and each 2-sector breakdown for indeterminacy for the closed-model without value added as inputs and GDP as outputs

\begin{tabular}{|c|c|c|c|c|c|c|c|c|c|}
\hline \multirow[t]{2}{*}{37} & \multirow[t]{2}{*}{12} & \multirow[t]{2}{*}{7} & \multicolumn{7}{|l|}{2} \\
\hline & & & Energy and food & Health & Govt. & Elec. and info. & Banking & Mfg. and const. & Business \\
\hline 1 & 0.46 & 0.14 & -0.15 & -0.044 & 0.19 & 0.31 & -0.18 & 0.37 & 0.081 \\
\hline- & 1 & 0.94 & -0.76 & 0.79 & -0.2 & 0.7 & 0.73 & -0.63 & 0.85 \\
\hline- & - & 1 & -0.83 & 0.87 & -0.21 & 0.68 & 0.91 & -0.85 & 0.96 \\
\hline- & - & - & 1 & -0.55 & -0.099 & -0.86 & -0.86 & 0.7 & -0.91 \\
\hline- & - & - & - & 1 & -0.61 & 0.31 & 0.76 & -0.79 & 0.75 \\
\hline- & - & - & - & - & 1 & 0.27 & -0.14 & 0.23 & -0.018 \\
\hline- & - & - & - & - & - & 1 & 0.65 & -0.51 & 0.72 \\
\hline- & - & - & - & - & - & - & 1 & -0.95 & 0.94 \\
\hline- & - & - & - & - & - & - & - & 1 & -0.86 \\
\hline- & - & - & - & - & - & - & - & - & 1 \\
\hline
\end{tabular}

and 7-sector aggregations. The 37-, 12-, and 7-sector aggregations are all highly positively correlated $(r>0.75)$ for all three information theory metrics (Tables 6, 8, 10) as well as the interpretive metrics (Tables 12, 14). The main exception is poor correlation for the 7-sector conditional entropy.

Many 2-sector Use table aggregations also show high correlation $(|r|>0.75)$ to the 37-sector model (see top row of correlations). All are highly correlated for mutual constraint. The exceptions for indeterminacy are the "Health" and "Banking" 2-sector models. The only 2-sector aggregations with high correlation for conditional entropy are "Manufacturing and Construction" and "Government." All of the interpretive metrics show high correlation $|r|>0.9$ for the 2-sector Use table aggregations except for calculations of hierarchy and equality for the "Health" and "Banking" 2-sector models.

\section{Closed-Model Results (Intermediate Transactions Only)}

In contrast to open-model correlations, the reduced sector closed-model aggregations generally have less correlation to the 37-sector representation. The 12- and 7-sector aggregations are highly positively correlated $(|r|>0.75)$ with the 37-sector model for mutual constraint and conditional entropy, but not for indeterminacy. This result is interesting because indeterminacy is equal to mutual constraint plus conditional entropy-correlation of the parts does not equal correlation for the sum of the parts. Also, no 2-sector closed-model aggregations have high correlation to the 37-sector harmonization for indeterminacy (Table 5). These closed-model results indicate that indeterminacy alone might miss some significant structure of the economy as represented by the I-O tables. 
Table 6 Table showing correlations for 37-, 12-, 7-, and each 2-sector breakdown for indeterminacy for the open-model with value added as inputs and GDP as outputs

\begin{tabular}{|c|c|c|c|c|c|c|c|c|c|}
\hline \multirow[t]{2}{*}{37} & \multirow[t]{2}{*}{12} & \multirow[t]{2}{*}{7} & \multicolumn{7}{|l|}{2} \\
\hline & & & Energy and food & Health & Govt. & Elec. and info. & Banking & Mfg. and const. & Business \\
\hline 1 & 0.98 & 0.93 & 0.9 & 0.26 & 0.97 & 0.9 & -0.075 & 0.97 & 0.91 \\
\hline- & 1 & 0.97 & 0.91 & 0.35 & 0.96 & 0.91 & 0.014 & 0.96 & 0.92 \\
\hline- & - & 1 & 0.83 & 0.39 & 0.91 & 0.9 & 0.15 & 0.88 & 0.92 \\
\hline- & - & - & 1 & 0.42 & 0.93 & 0.71 & -0.12 & 0.96 & 0.75 \\
\hline- & - & - & - & 1 & 0.18 & 0.028 & -0.26 & 0.32 & 0.076 \\
\hline- & - & - & - & - & 1 & 0.89 & 0.052 & 0.97 & 0.92 \\
\hline- & - & - & - & - & - & 1 & 0.21 & 0.83 & 0.91 \\
\hline- & - & - & - & - & - & - & 1 & -0.13 & 0.21 \\
\hline- & - & - & - & - & - & - & - & 1 & 0.84 \\
\hline- & - & - & - & - & - & - & - & - & 1 \\
\hline
\end{tabular}

Table 7 Table showing correlations for 37-, 12-, 7-, and each 2-sector breakdown for mutual constraint for the closed-model without value added as inputs and GDP as outputs

\begin{tabular}{|c|c|c|c|c|c|c|c|c|c|}
\hline \multirow[t]{2}{*}{37} & \multirow[t]{2}{*}{12} & \multirow[t]{2}{*}{7} & \multicolumn{7}{|l|}{2} \\
\hline & & & Energy and food & Health & Govt. & Elec. and info. & Banking & Mfg. and const. & Business \\
\hline 1 & 0.98 & 0.86 & 0.93 & 0.24 & 0.34 & -0.92 & -0.8 & 0.93 & -0.73 \\
\hline- & 1 & 0.9 & 0.96 & 0.18 & 0.28 & -0.87 & -0.86 & 0.94 & -0.71 \\
\hline- & - & 1 & 0.89 & 0.12 & 0.17 & -0.68 & -0.77 & 0.84 & -0.5 \\
\hline- & - & - & 1 & 0.035 & 0.2 & -0.87 & -0.84 & 0.9 & -0.59 \\
\hline- & - & - & - & 1 & 0.82 & -0.29 & -0.37 & 0.3 & -0.46 \\
\hline- & - & - & - & - & 1 & -0.48 & -0.45 & 0.43 & -0.59 \\
\hline- & - & - & - & - & - & 1 & 0.71 & -0.85 & 0.7 \\
\hline- & - & - & - & - & - & - & 1 & -0.89 & 0.74 \\
\hline- & - & - & - & - & - & - & - & 1 & -0.8 \\
\hline- & - & - & - & - & - & - & - & - & 1 \\
\hline
\end{tabular}

For mutual constraint (Table 7), two of the 2-sector aggregations are significantly positively correlated ("Energy and Food" and "Manufacturing and Construction"), while two are significantly negatively correlated ("Electronics and Information" and "Banking"). Table 2 and Fig. 4 show that closed-model 37-sector mutual constraint generally decreased until 2002 (along with increasing power consumption) before increasing to 2012. The shares of intermediate transactions for both "Energy and Food" and "Manufacturing and Construction" decreased through 2002, and their 2-sector representations also decreased in mutual constraint. On the other hand, the 2-sector "Electronics and Information" aggregation increased mutual constraint from the 1960s through 2002, and the "Banking" aggregation increased mutual constraint primarily from 1947 through 2007 (1982 being somewhat of an anomaly) before declining to 2012 after the financial crisis and Great Recession.
Considering conditional entropy for the closed-model configuration (Table 9), there are no 2-sector aggregations that are highly correlated $(|r|>0.75)$ with the 37 -sector harmonization. However, the signs of the correlation coefficients are generally opposite that of mutual constraint, indicating that (at least since 1958) conditional entropy and mutual constraint generally moved in opposite directions. Further, Fig. 8d indicates a clear rise in (closed-model) conditional entropy as $\mathrm{NPR}_{\text {economic }}$ increases, and vice versa for 2002-2012. Thus, another indicator that cheaper resources (energy and food) translated to more distributed flows among economic sectors (and vice versa).

The closed-model correlations for efficiency and redundancy (the correlations are identical as the metrics are defined as opposites) are similar in trend to that of mutual constraint (Table 13; Fig. 9a). The 2-sector aggregations for "Energy and food," "Electronics and Information," 
Table 8 Table showing correlations for 37-, 12-, 7-, and each 2-sector breakdown for mutual constraint for the open-model with value added as inputs and GDP as outputs

\begin{tabular}{|c|c|c|c|c|c|c|c|c|c|}
\hline \multirow[t]{2}{*}{37} & \multirow[t]{2}{*}{12} & \multirow[t]{2}{*}{7} & \multicolumn{7}{|l|}{2} \\
\hline & & & Energy and food & Health & Govt. & Elec. and info. & Banking & Mfg. and const. & Business \\
\hline 1 & 0.99 & 0.99 & 0.99 & 0.99 & 0.99 & 0.97 & 0.97 & 0.99 & 0.98 \\
\hline- & 1 & 1 & 0.99 & 0.99 & 0.99 & 0.98 & 0.97 & 0.99 & 0.99 \\
\hline- & - & 1 & 1 & 0.99 & 0.99 & 0.99 & 0.98 & 0.99 & 0.99 \\
\hline- & - & - & 1 & 0.99 & 0.99 & 0.98 & 0.97 & 0.99 & 1 \\
\hline- & - & - & - & 1 & 1 & 0.99 & 0.99 & 1 & 1 \\
\hline- & - & - & - & - & 1 & 0.99 & 0.99 & 1 & 1 \\
\hline- & - & - & - & - & - & 1 & 0.98 & 0.99 & 0.99 \\
\hline- & - & - & - & - & - & - & 1 & 0.98 & 0.99 \\
\hline- & - & - & - & - & - & - & - & 1 & 1 \\
\hline- & - & - & - & - & - & - & - & - & 1 \\
\hline
\end{tabular}

Table 9 Table showing correlations for 37-, 12-, 7-, and each 2-sector breakdown for conditional entropy for the closed-model without value added as inputs and GDP as outputs

\begin{tabular}{|c|c|c|c|c|c|c|c|c|c|}
\hline \multirow[t]{2}{*}{37} & \multirow[t]{2}{*}{12} & \multirow[t]{2}{*}{7} & \multicolumn{7}{|l|}{2} \\
\hline & & & Energy and food & Health & Govt. & Elec. and info. & Banking & Mfg. and const. & Business \\
\hline 1 & 0.89 & 0.79 & -0.56 & 0.56 & -0.1 & 0.65 & 0.48 & -0.3 & 0.7 \\
\hline- & 1 & 0.98 & -0.66 & 0.82 & -0.23 & 0.61 & 0.79 & -0.69 & 0.9 \\
\hline- & - & 1 & -0.7 & 0.86 & -0.22 & 0.57 & 0.88 & -0.8 & 0.95 \\
\hline- & - & - & 1 & -0.38 & -0.2 & -0.77 & -0.8 & 0.6 & -0.84 \\
\hline- & - & - & - & 1 & -0.62 & 0.13 & 0.72 & -0.74 & 0.72 \\
\hline- & - & - & - & - & 1 & 0.4 & -0.1 & 0.21 & 0.012 \\
\hline- & - & - & - & - & - & 1 & 0.52 & -0.35 & 0.64 \\
\hline- & - & - & - & - & - & - & 1 & -0.95 & 0.93 \\
\hline- & - & - & - & - & - & - & - & 1 & -0.83 \\
\hline- & - & - & - & - & - & - & - & - & 1 \\
\hline
\end{tabular}

and "Manufacturing and Construction" show high correlation (positive, negative, positive, respectively) to the $37-$, 12-, and 7-sector intermediate Use table aggregations. Also, overall the correlations for any given aggregation (e.g., along a column) are much more consistent for efficiency and redundancy. This suggests that Ulanowicz's metric that I use for efficiency (Eq. 21) is perhaps a more consistent measure of system balance toward efficiency than mutual constraint alone.

The closed-model correlations for equality and hierarchy are much less consistent (Table 11; Fig. 9b). The 12and 7-sector correlations are poor both between each other and to the 37-sector harmonization. The 7-sector aggregation is negatively correlated with that of the 37-sector. Thus, it is difficult to interpret any meaning from the 2-sector correlations to the 37-, 12-, and 7-sector versions, or among themselves. These results pose questions for future research in terms of understanding the equality of monetary flows through the sectors when defining both the number of sectors and which to aggregate.

\section{Scaling Laws of U.S. Use Tables: Phase Space}

Here I investigate whether some simple scaling laws might describe the U.S. Use tables. To test this, I generated many sample data for a $37 \times 37$ matrix-based upon power laws and exponential distributions (with many randomly chosen scaling exponents) as well as randomly selecting matrix entries from a uniform distribution. Each matrix with sample data represents one marker in Figs. 10 and 11 that show results with blue dots and red dots representing randomized power and exponential law-generated matrix 
Table 10 Table showing correlations for 37-, 12-, 7-, and each 2-sector breakdown for conditional entropy for the open-model with value added as inputs and GDP as outputs

\begin{tabular}{|c|c|c|c|c|c|c|c|c|c|}
\hline \multirow[t]{2}{*}{37} & \multirow[t]{2}{*}{12} & \multirow[t]{2}{*}{7} & \multicolumn{7}{|l|}{2} \\
\hline & & & Energy and food & Health & Govt. & Elec. and info. & Banking & Mfg. and const. & Business \\
\hline 1 & 0.86 & 0.67 & 0.65 & -0.51 & 0.75 & 0.71 & -0.67 & 0.85 & 0.34 \\
\hline- & 1 & 0.93 & 0.4 & -0.17 & 0.56 & 0.73 & -0.34 & 0.55 & 0.55 \\
\hline- & - & 1 & 0.25 & -0.028 & 0.52 & 0.69 & -0.082 & 0.38 & 0.65 \\
\hline- & - & - & 1 & -0.39 & 0.72 & 0.2 & -0.76 & 0.9 & -0.16 \\
\hline- & - & - & - & 1 & -0.75 & -0.61 & 0.25 & -0.55 & -0.32 \\
\hline- & - & - & - & - & 1 & 0.69 & -0.42 & 0.82 & 0.45 \\
\hline- & - & - & - & - & - & 1 & -0.062 & 0.45 & 0.67 \\
\hline- & - & - & - & - & - & - & 1 & -0.81 & 0.3 \\
\hline- & - & - & - & - & - & - & - & 1 & 0.013 \\
\hline- & - & - & - & - & - & - & - & - & 1 \\
\hline
\end{tabular}

Table 11 Table showing correlations for 37-, 12-, 7-, and each 2-sector breakdown for hierarchy and equality for the closed-model without value added as inputs and GDP as outputs

\begin{tabular}{llllllllll}
\hline 37 & 12 & 7 & 2 & & & & \\
\cline { 3 - 8 } & & & Energy and food & Health & Govt. & Elec. and info. & Banking & Mfg. and const. & Business \\
\hline 1 & 0.26 & -0.59 & 0.49 & -0.64 & 0.37 & -0.36 & -0.77 & 0.87 & -0.6 \\
- & 1 & 0.58 & -0.48 & 0.48 & -0.048 & 0.51 & 0.29 & -0.17 & 0.46 \\
- & - & 1 & -0.88 & 0.89 & -0.21 & 0.75 & 0.94 & -0.89 & 0.96 \\
- & - & - & 1 & -0.63 & -0.042 & -0.9 & -0.89 & 0.76 & -0.93 \\
- & - & - & - & 1 & -0.6 & 0.45 & 0.8 & -0.83 & 0.78 \\
- & - & - & - & - & 1 & 0.16 & -0.16 & 0.25 & -0.045 \\
- & - & - & - & - & - & 1 & 0.73 & -0.63 & 0.77 \\
- & - & - & - & - & - & - & -0.95 & 0.95 \\
- & - & - & - & - & - & - & - & -0.89 \\
- & - & - & - & - & - & - & - & 1 \\
\hline
\end{tabular}

entries, respectively. Supplemental Figure S6 shows a comparison of the phase space the 37-, 12-, and 7-sector models of the Use tables.

\section{Phase Space for Closed-Model of Use I-O Tables}

In Fig. 10, the distribution of intermediate transactions (closed-model) within the U.S. economy, as represented by the Use tables, can be approximated by a power law from 1958 to approximately 1982. The first year with I-O table data (1947) lies outside of the blue-dotted (power law) region, and the same holds for the last 6 benchmark years with data (1987 to 2012). This is more clearly seen in the zoomed view of Fig. 10b. Where the Use table information theory calculations reside near the Monte Carlo simulations of power law-generated entries (1958-1982), the $i^{\text {th }}$ entries of the $37 \times 37$ matrix are scaled as $T_{i}=i^{-k}$ with $0.9<k<1$. After 1982 , the phase space location of the intermediate transactions moves away (to the left) from this power law boundary.

The results from filling each $\mathrm{I}-\mathrm{O}$ entry with a random number from a uniform distribution are in a very tight range (not distinguishable from the other results) where labeled "Uniform" near the bounding case of a uniform matrix at maximum conditional entropy and zero mutual constraint. The I-O table calculations do not appear near the trends for any of the Monte Carlo I-O matrix entries generated from exponential distributions.

\section{Phase Space for Open-Model of Use I-O Tables}

Unlike calculations for the closed-model for bounding cases of uniform and diagonal matrices, each does not have a single bounding value for the open network model. This is because one must make assumptions about how the sectoral inputs and outputs are distributed among all 
Table 12 Table showing correlations for 37-, 12-, 7-, and each 2-sector breakdown for hierarchy and equality for the open-model with value added as inputs and GDP as outputs

\begin{tabular}{|c|c|c|c|c|c|c|c|c|c|}
\hline \multirow[t]{2}{*}{37} & \multirow[t]{2}{*}{12} & \multirow[t]{2}{*}{7} & \multicolumn{7}{|l|}{2} \\
\hline & & & Energy and food & Health & Govt. & Elec. and info. & Banking & Mfg. and const. & Business \\
\hline 1 & 0.99 & 0.97 & 0.95 & 0.78 & 0.99 & 0.95 & 0.74 & 0.99 & 0.98 \\
\hline- & 1 & 0.99 & 0.96 & 0.83 & 0.99 & 0.95 & 0.78 & 0.99 & 0.98 \\
\hline- & - & 1 & 0.93 & 0.83 & 0.97 & 0.95 & 0.83 & 0.95 & 0.98 \\
\hline- & - & - & 1 & 0.86 & 0.96 & 0.85 & 0.7 & 0.97 & 0.91 \\
\hline- & - & - & - & 1 & 0.76 & 0.67 & 0.55 & 0.81 & 0.73 \\
\hline- & - & - & - & - & 1 & 0.95 & 0.78 & 0.99 & 0.98 \\
\hline- & - & - & - & - & - & 1 & 0.81 & 0.92 & 0.95 \\
\hline- & - & - & - & - & - & - & 1 & 0.71 & 0.81 \\
\hline- & - & - & - & - & - & - & - & 1 & 0.95 \\
\hline- & - & - & - & - & - & - & - & - & 1 \\
\hline
\end{tabular}

Table 13 Table showing correlations for 37-, 12-, 7-, and each 2-sector breakdown for efficiency and redundancy for the closed-model without value added as inputs and GDP as outputs

\begin{tabular}{llllllllll}
\hline 37 & 12 & 7 & 2 & & & & \\
\cline { 3 - 8 } & & & Energy and food & Health & Govt. & Elec. and info. & Banking & Mfg. and const. & Business \\
\hline 1 & 1 & 0.99 & 0.93 & 0.4 & 0.34 & -0.83 & -0.43 & 0.75 & -0.51 \\
- & 1 & 1 & 0.94 & 0.38 & 0.33 & -0.83 & -0.44 & 0.75 & -0.5 \\
- & - & 1 & 0.93 & 0.37 & 0.35 & -0.85 & -0.4 & 0.73 & -0.49 \\
- & - & - & 1 & 0.41 & 0.36 & -0.79 & -0.49 & 0.8 & -0.41 \\
- & - & - & - & 1 & 0.89 & -0.62 & -0.41 & 0.37 & -0.63 \\
- & - & - & - & - & 1 & -0.61 & -0.34 & 0.31 & -0.68 \\
- & - & - & - & - & - & 1 & 0.16 & -0.5 & 0.61 \\
- & - & - & - & - & - & - & 1 & -0.72 & 0.45 \\
- & - & - & - & - & - & - & - & -0.44 \\
- & - & - & - & - & - & - & - & 1 \\
\hline
\end{tabular}

sectors (see Fig. 11). For each distribution (e.g., exponential or power law) used to create Monte Carlo inputs of the intermediate I-O matrix, I use the same distribution to estimate the 37 input values that mimic value added in the I-O framework. For uniform and diagonal cases, each nonzero intermediate matrix entry is equal and each input (and output) entry is equal. In keeping with economic I-O accounting conventions, I then solve for the system outputs as a function of the input (=value added) and intermediate matrix values. That is to say, for any given $i^{\text {th }}$ row and column, the sum of all rows for column $i$ plus input $i$ must equal the sum of all columns for row $i$ plus output $i$ (Miller and Blair 2009). Thus, for uniform and diagonal distributions, each input (= each output) value generally does not equal the value placed in the intermediate matrix.

The results are similar to that of the closed-model in that for the early years (e.g., 1958 and 1963), the open-model I$O$ results reside near the range of some power law distributions, but not near those for a uniform matrix, diagonal matrix, or exponential law-driven entries. One can see after 1967 (the fourth data point) the calculations move down and to the left in the phase space, whereas in the closed-model the trend is still up and to the left.

\section{Scaling Laws for Use Tables: Logarithmic Plots}

Figure 12 shows six semilogarithmic plots to further explore the possible scaling laws of the U.S. Use tables. Each of the subfigures indicates one of three types of results for either the closed-model (Fig. 12a, c, e) or open-model (Fig. 12b, d, f) representation of the economy. The $y$-axis of each subplot of Fig. 12 is one minus the cumulative fraction of flows, $F(t)$, considered when summing the fraction of all flows in order from the smallest to largest entry.

If the trends in Fig. 12 follow a straight line, this implies power law scaling. If all of the flows are equal (e.g., full 
Table 14 Table showing correlations for 37-, 12-, 7-, and each 2-sector breakdown for efficiency and redundancy for the open-model with value added as inputs and GDP as outputs

\begin{tabular}{|c|c|c|c|c|c|c|c|c|c|}
\hline \multirow[t]{2}{*}{37} & \multirow[t]{2}{*}{12} & \multirow[t]{2}{*}{7} & \multicolumn{7}{|l|}{2} \\
\hline & & & Energy and food & Health & Govt. & Elec. and info. & Banking & Mfg. and const. & Business \\
\hline 1 & 0.99 & 0.99 & 0.98 & 0.96 & 0.96 & 0.95 & 0.92 & 0.97 & 0.96 \\
\hline- & 1 & 1 & 0.99 & 0.96 & 0.97 & 0.97 & 0.93 & 0.98 & 0.98 \\
\hline- & - & 1 & 0.99 & 0.97 & 0.98 & 0.97 & 0.94 & 0.99 & 0.98 \\
\hline- & - & - & 1 & 0.98 & 0.99 & 0.97 & 0.95 & 0.99 & 0.99 \\
\hline- & - & - & - & 1 & 0.97 & 0.93 & 0.93 & 0.98 & 0.95 \\
\hline- & - & - & - & - & 1 & 0.98 & 0.98 & 0.99 & 0.99 \\
\hline- & - & - & - & - & - & 1 & 0.96 & 0.97 & 0.99 \\
\hline- & - & - & - & - & - & - & 1 & 0.94 & 0.97 \\
\hline- & - & - & - & - & - & - & - & 1 & 0.98 \\
\hline- & - & - & - & - & - & - & - & - & 1 \\
\hline
\end{tabular}

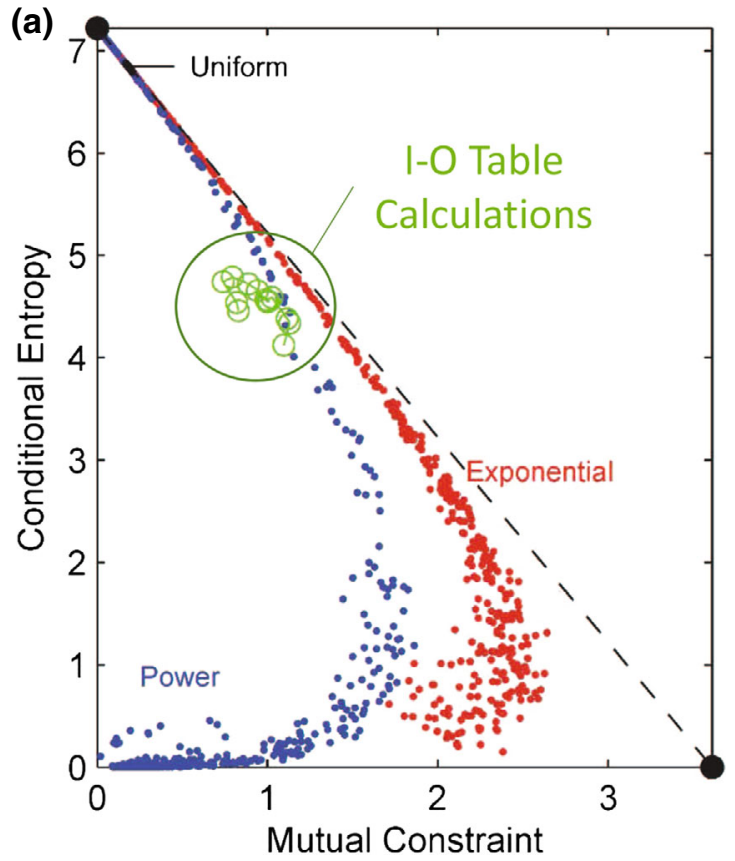

Fig. 10 A plot of the 37-sector harmonized U.S. Use I-O matrix (closed-model: without value added as inputs and GDP as outputs) information theory trends along with results from generating multiple $37 \times 37$ matrices where entries are governed by a power law and then randomly distributed (blue dots), an exponential law and then randomly distributed (red dots), or a random values generated from a uniform distribution (black dots highly concentrated where labeled

node flow equality), then this plot will appear as a vertical line. Thus, for Fig. 12a-b, the closer is the plotted line to a vertical line (e.g., the steeper the slope), the more equal is the equality metric. Note that equality is highest for the closed-model in 1967 and for the open-model in 1958. (b)

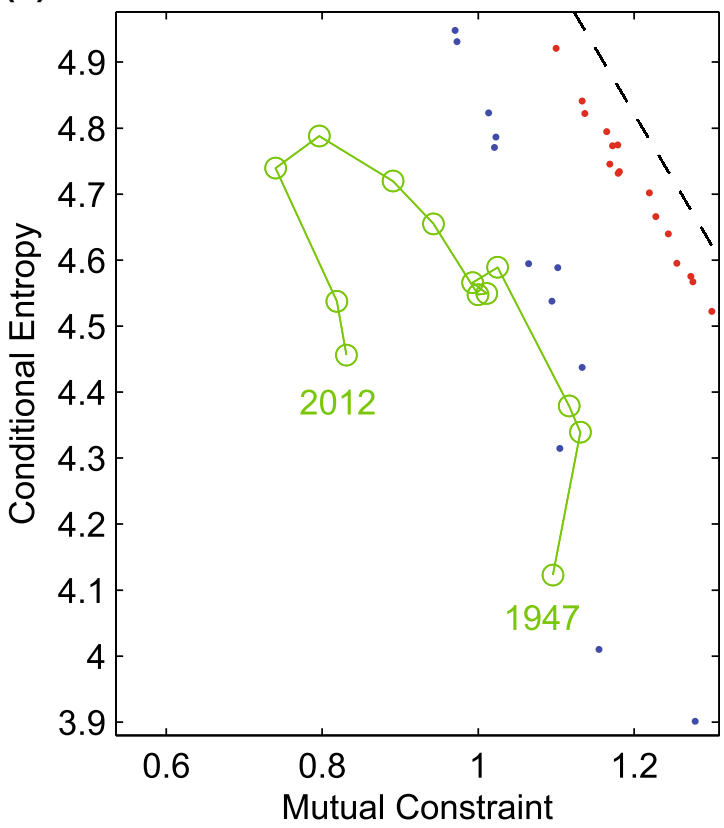

'Uniform'). The dashed lines represent upper bounds. a Results at full extent. b A zoomed view focusing on the areas with the calculations for 37 -sector harmonized U.S. Use tables. The U.S. Use tables from 1958 to 1982 reside near a power law scaling (where the $i$ th entries are scaled as $T_{i}=i^{-k}, 0.9<k<1$ ) but diverge from power law scaling after 1982

In each subsection of this section, I also show a table of the top ten largest plotted values in order to indicate which sectors are responsible for the largest economic flows each year. The largest economic flows in turn indicate larger mathematical importance in terms of scaling laws and the information theory calculations. 
(a)

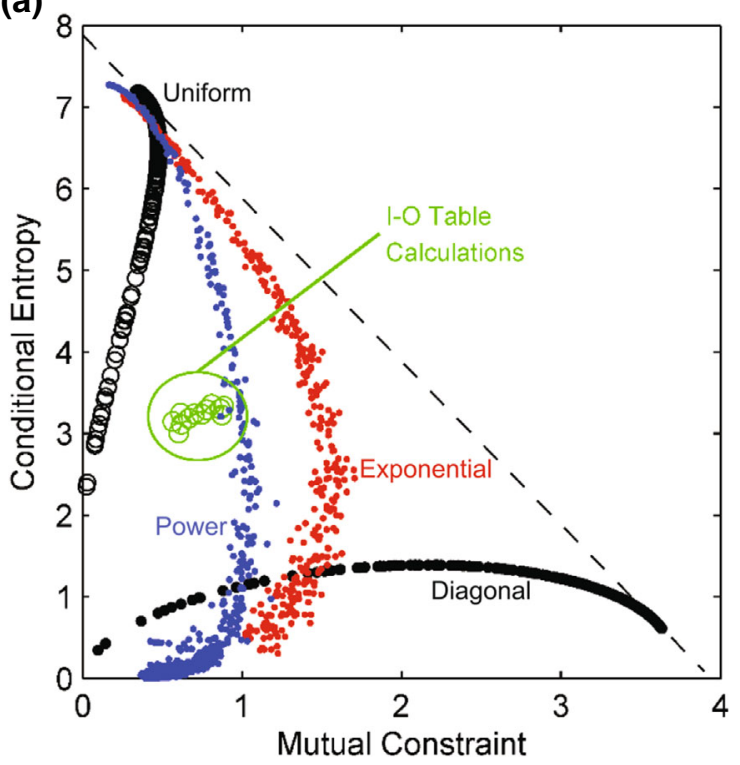

Fig. 11 A conditional entropy versus mutual constraint phase space plot of the information theory calculations for the harmonized 37sector U.S. $\left(\Psi_{\max }=7.8842\right.$ and $\left.X_{\max }=\frac{\Psi_{\max }}{2}\right)$. Use I-O tables when including sectoral value added as inputs and sectoral GDP as outputs (green circles connected by line). All Monte Carlo calculations use a $37 \times 37$ matrix. The dashed lines represent upper bounds. a All calculations. Open black circles uniform matrices with uniform inputs

\section{Considering Each Use Table Entry}

Figure 12a shows the cumulative faction of all monetary flows in the 37-sector harmonized Use table when considering only the intermediate transactions (or closedmodel). Figure $12 \mathrm{~b}$ shows the same when considering the open-model where each sectoral input and output flow is treated as another single entry. By visual inspection, the closed-model trends of more closely approximate a line (e.g., power law) for the middle range of years (1958-1977) than the open-model results. This observation is confirms that of Figs. 10 and 11.

Table 15 lists the sectors involved in each of the top ten largest individual intermediate purchases (e.g., individual entries in the $\mathrm{I}-\mathrm{O}$ table). The two harmonized food sectors have one to three of the top ten purchases each year, and the energy sectors have a single top ten purchase in each of 1982, 1997, 2007, and 2012. One of the major trends is that the harmonized sector Food Products, Stores, and Services $(=18)$ effectively replaces the Farms $(=1)$ sector as the sector with the largest single sectoral purchase, and farms drops out of the top ten purchases after 1987 after having the single largest intermediate purchase for all prior years. This change in influence from the Farms $(=1)$ sector happens before the change to NAICS in 1997. The Petroleum and Coal Products (=23) (b)

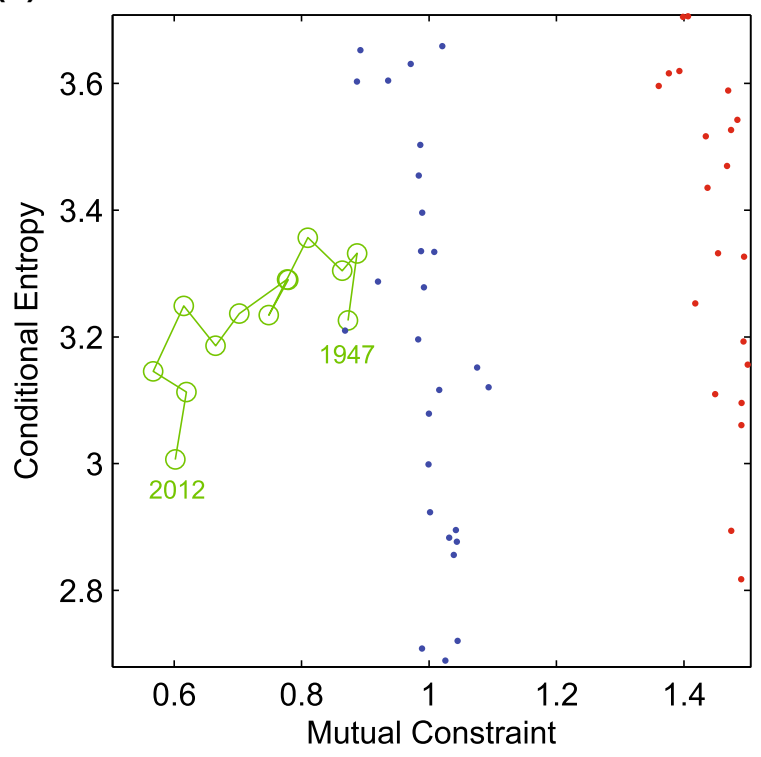

and outputs at various scaling (sum of inputs and outputs relative to intermediate transactions); closed black circles diagonal matrices results run along bottom edge of middle and right plots; red dots exponential matrices; blue dots power law matrices. b A zoomed view to the locations of the calculations of the 37-sector harmonized U.S. Use tables

sector has one of the top ten purchases in each benchmark year, rising from rank 7-10 in 1987 and earlier to rank 2-5 from 1992 and later. Each year this largest purchase by the Petroleum and coal products sector is (unsurprisingly) for primary energy feedstock from the Oil and Gas Extraction (=3) sector.

\section{Summing Entries by Sales of Each Sector}

In Fig. 12c, d, I sum all 37 sales from a given sector to all 37 sectors (e.g., sum all column entries for a given row, $i$ ) and plot the cumulative distribution of these sums. The trends for 1997-2012 appear generally different from the other years, and this is possibly due to effects from the underlying table constructions (i.e., using the "before redefinitions" Use tables organized by NAICS for 1997 and later versus by SIC for 1992 and earlier).

Table 16 lists the top ten sectors for total intermediate sales each year. Both harmonized food sectors have top ten sales from 1947 to 1982 (with Farms (=1) having the highest sales each of those years). From 1987 to 2012, the Farms sector is no longer in the top ten, but Food Products, Stores, and Services $(=18)$ is within the top four. For the energy sectors, Utilities (=5) had the highest or second highest sales for 1977, 1982, and 1992, but does not appear in the top ten otherwise. The Petroleum and Coal Products 
(a)

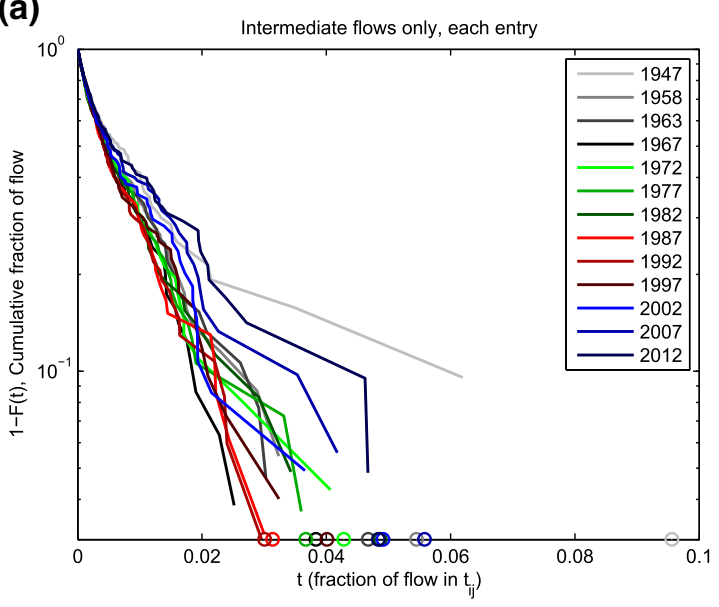

(c)

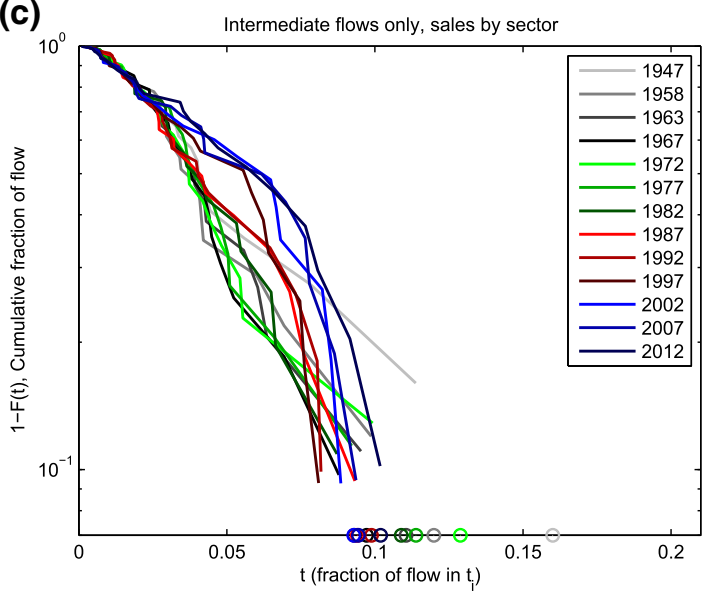

(e)

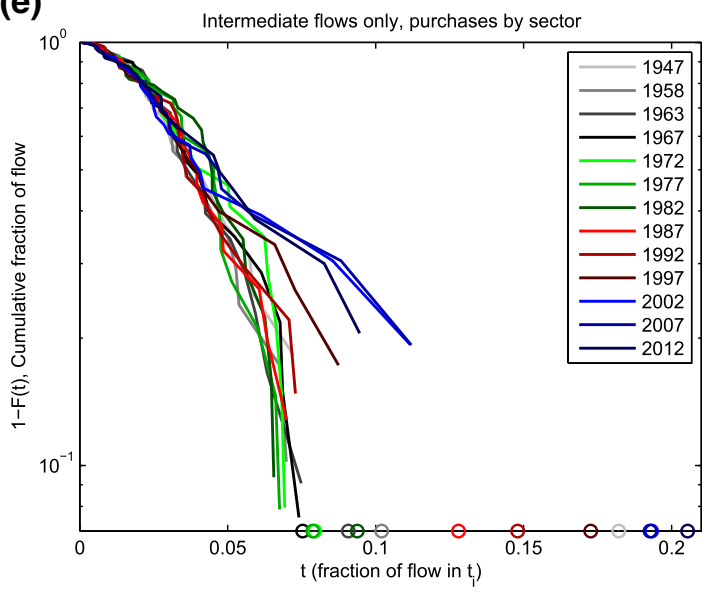

Fig. 12 Semilogarithmic plots of 37-sector harmonized IO tables as the cumulative fraction of flows, either the relative size of each entry or the relative sum of entries for a sector ( $y$-axis) versus the fraction of the total sum of that entry or sum ( $x$-axis). The circles along the $x$-axis represent the final (and largest) value of the IO table or sum (as this final value cannot be plotted on logarithmic axis). a Individual intermediate (only) IO entries, b Individual intermediate IO entries (b)

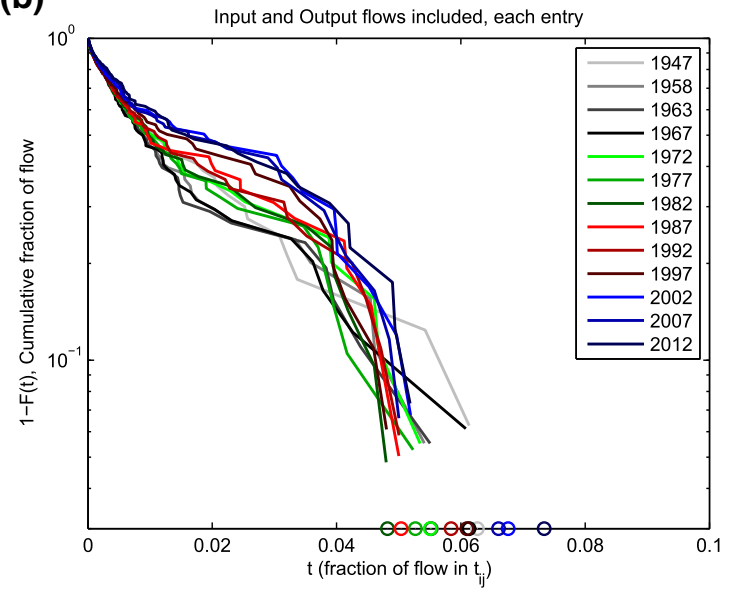

(d) Input and Output flows included, sales by sector

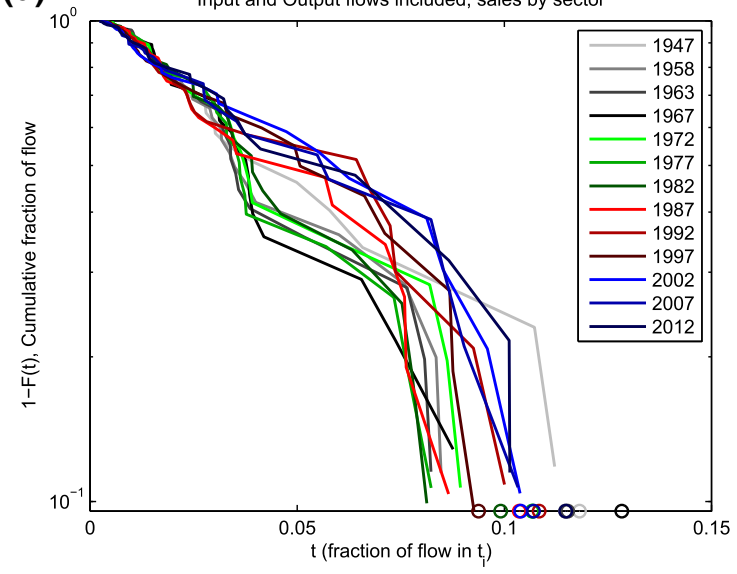

(f)

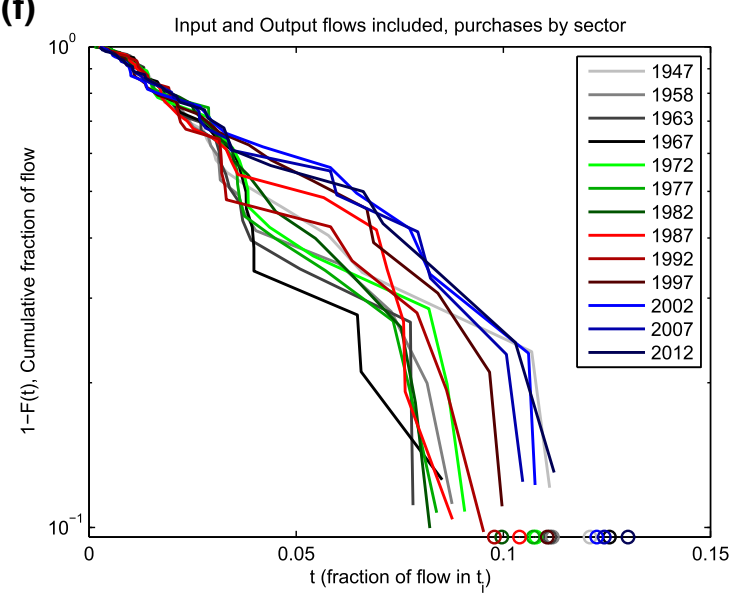

with inputs and output flows also, c sum of the sales (each row) considering intermediate IO entries only, $\mathbf{d}$ sum of the purchases (each column) considering intermediate IO entries only, e sum of the sales (each row) considering intermediate IO entries and output (GDP-imports) flows, f sum of the purchases (each column) considering intermediate IO entries and input (value added + imports) flows 
Table 15 Table showing the ten entries with the highest individual intermediate economic flows for each year (largest entry at top of table)

\begin{tabular}{lllllllllllll}
\hline 1947 & 1958 & 1963 & 1967 & 1972 & 1977 & 1982 & 1987 & 1992 & 1997 & 2002 & 2007 & 2012 \\
\hline 1 & 1 & 1 & 1 & 1 & 1 & 1 & 1 & 6 & 12 & 14 & 18 & 18 \\
9 & 6 & 6 & 6 & 6 & 6 & $\mathbf{5}$ & 6 & 18 & 14 & 18 & $\mathbf{2 3}$ & 18 \\
14 & 6 & 9 & 6 & 6 & 9 & $\mathbf{5}$ & 6 & 18 & 18 & $\mathbf{2 3}$ & 24 & $\mathbf{2 3}$ \\
18 & 9 & 10 & 9 & 9 & 10 & 6 & 14 & $\mathbf{2 3}$ & 18 & 26 & 26 & 24 \\
18 & 10 & 14 & 10 & 10 & 14 & 6 & 18 & 26 & $\mathbf{2 3}$ & 29 & 30 & 26 \\
19 & 14 & 18 & 14 & 14 & 18 & 18 & 18 & 30 & 24 & 30 & 31 & 30 \\
20 & 18 & 18 & 18 & 18 & 18 & 18 & $\mathbf{2 3}$ & 31 & 26 & 31 & 31 & 31 \\
20 & 18 & 19 & 18 & 18 & $\mathbf{2 3}$ & $\mathbf{2 3}$ & 26 & 31 & 30 & 31 & 32 & 32 \\
$\mathbf{2 3}$ & 19 & $\mathbf{2 3}$ & $\mathbf{2 3}$ & $\mathbf{2 3}$ & 26 & 26 & 30 & 33 & 31 & 33 & 33 & 33 \\
32 & $\mathbf{2 3}$ & 32 & 26 & 31 & 31 & 31 & 31 & 33 & 31 & 33 & 33 & 33
\end{tabular}

The numbers indicate which of the 37 sectors made that individual purchase, or sector $i$ making purchase $T_{i j}$ where $i=j$ is possible. The food sectors (numbers italicized in table) are 1 (Farms) and 18 (Food products, Stores, and Services), and the energy sectors (numbers bold in table) are 3 (Oil and gas extraction), 5 (Utilities), and 23 (Petroleum and coal products)
Table 16 Table showing the ten sectors with the highest sum of total intermediate sales for each year (largest entry at top of table)

\begin{tabular}{lllllllllllll}
\hline 1947 & 1958 & 1963 & 1967 & 1972 & 1977 & 1982 & 1987 & 1992 & 1997 & 2002 & 2007 & 2012 \\
\hline 1 & 1 & 1 & 1 & 1 & 1 & 1 & 6 & $\mathbf{5}$ & 6 & 6 & 6 & 6 \\
6 & 6 & 6 & 6 & 6 & $\mathbf{5}$ & $\mathbf{5}$ & 14 & 6 & 12 & 14 & 14 & 14 \\
9 & 9 & 9 & 9 & 9 & 6 & 6 & 18 & 14 & 14 & 18 & 18 & 18 \\
14 & 10 & 10 & 11 & 11 & 9 & 11 & $\mathbf{2 3}$ & 18 & 18 & 24 & $\mathbf{2 3}$ & $\mathbf{2 3}$ \\
18 & 11 & 11 & 14 & 14 & 11 & 18 & 26 & 26 & 24 & 26 & 24 & 24 \\
19 & 14 & 14 & 18 & 18 & 14 & $\mathbf{2 3}$ & 28 & 28 & 26 & 29 & 26 & 26 \\
20 & 18 & 18 & 24 & 26 & 18 & 26 & 30 & 30 & 30 & 30 & 30 & 30 \\
26 & $\mathbf{2 3}$ & 26 & 26 & 31 & $\mathbf{2 3}$ & 28 & 31 & 31 & 31 & 31 & 31 & 31 \\
28 & 26 & 30 & 30 & 32 & 26 & 31 & 32 & 32 & 32 & 32 & 32 & 32 \\
32 & 32 & 32 & 32 & 33 & 33 & 33 & 33 & 33 & 33 & 33 & 33 & 33 \\
\hline
\end{tabular}

The numbers indicate which of the 37 sectors made those total sales (including to its own sector). The food sectors (numbers italicized in table) are 1 (Farms) and 18 (Food products, Stores, and Services), and the energy sectors (numbers bold in table) are 3 (Oil and gas extraction), 5 (Utilities), and 23 (Petroleum and coal products)
(=23) sector had the fourth highest sales for 1987, 2007, and 2012 and appeared in the top ten in 1958, 1977, and 1982. The other dominant sectors for sales, besides food and energy, are Construction (=6), Motor Vehicles (=14), Wholesale and Retail Trade $(=26)$, Business services $(=30)$, Insurance and banking $(=31)$, Real estate $(=32)$, and Health $(=33)$.

\section{Summing Entries by Purchases by Each Sector}

In Fig. 12e, f, I sum all 37 purchases of a given sector from all 37 sectors (e.g., sum all row entries for a given column, $i$ ) and plot the cumulative distribution of these sums. The distributions from 1997 to 2012 are visually different than before 1997, again indicating perhaps some underlying influence from the underlying change from SIC to NAICS in 1997. Consistent with the phase space trends in Figs. 10 and 11 the earlier years show scaling closer to a power law, but not the latter years. The scaling is not expected to be the same for the case of sum of sales (Fig. 12c, d) as compared to the case of the sum of purchases. For any given sector, the underlying BEA Use table data (NAICS, before redefinitions) do not have the sum of sales (all columns) equal to the sum of purchases (all rows).

Table 17 lists the top ten sectors for total intermediate purchases each year. In all years, the two food sectors are within the top six highest purchases from 1947 to 1992, with Farms $(=1)$ the top sector during that time span. From 1997 onward, the Food Products, Stores, and Services $(=18)$ sector has the third highest purchases and the Farm sector is no longer in the top ten (possibly due to redefinition of sectors from SIC to NAICS).

The energy sectors within the top ten highest purchases have an interesting pattern. The Petroleum and Coal Products $(=23)$ sector, responsible for refining oil into fuels, had the sixth highest purchases in 1982 and third 
Table 17 Table showing the ten sectors with the highest sum of total intermediate purchases for each year (largest entry at top of table)

\begin{tabular}{lllllllllllll}
\hline 1947 & 1958 & 1963 & 1967 & 1972 & 1977 & 1982 & 1987 & 1992 & 1997 & 2002 & 2007 & 2012 \\
\hline 1 & 1 & 1 & 1 & 1 & 1 & 1 & 1 & 1 & 10 & 10 & $\mathbf{3}$ & $\mathbf{3}$ \\
6 & 9 & 9 & 9 & $\mathbf{5}$ & $\mathbf{3}$ & $\mathbf{3}$ & $\mathbf{5}$ & $\mathbf{5}$ & 12 & 14 & 10 & 9 \\
9 & 10 & 10 & 10 & 9 & $\mathbf{5}$ & $\mathbf{5}$ & 9 & 6 & 18 & 18 & 18 & 18 \\
10 & 18 & 11 & 11 & 10 & 9 & 9 & 10 & 10 & 24 & 24 & $\mathbf{2 3}$ & $\mathbf{2 3}$ \\
18 & 24 & 18 & 18 & 18 & 10 & 18 & 18 & 18 & 26 & 26 & 24 & 24 \\
19 & 26 & 24 & 24 & 26 & 18 & $\mathbf{2 3}$ & 26 & 26 & 28 & 28 & 26 & 26 \\
26 & 28 & 26 & 26 & 28 & 26 & 26 & 28 & 28 & 29 & 29 & 28 & 28 \\
28 & 30 & 28 & 28 & 30 & 28 & 28 & 30 & 30 & 30 & 30 & 30 & 30 \\
30 & 31 & 30 & 30 & 31 & 30 & 30 & 31 & 31 & 31 & 31 & 31 & 31 \\
32 & 32 & 32 & 32 & 32 & 32 & 32 & 32 & 32 & 32 & 32 & 32 & 32
\end{tabular}

The numbers indicate which of the 37 sectors made the total purchases (including from its own sector). The food sectors (numbers italicized in table) are 1 (Farms) and 18 (Food products, Stores, and Services), and the energy sectors (numbers bold in table) are 3 (Oil and gas extraction), 5 (Utilities), and 23 (Petroleum and coal products) highest in both 2007 and 2012, but is not within the top ten otherwise. In the time span from 1972 to 1992, Utilities (=5) had the second or third highest total purchases, but was not within the top ten in any other years. The Oil and Gas Extraction sector (=3) had the second highest purchases in 1977 and 1982 and the highest purchases in 2007 and 2012. This trend reflects the two time periods with significant investment in oil and gas extraction. The year 1982 is unique in having all five of the energy and food sectors within the top ten for total purchases.

\section{Discussion}

\section{Interpretation of Trends}

There are three general insights from analyzing the trends of the information theory metrics. First, for the 37-sector model three phases are defined by distinct combinations of directional change for the information theory metrics that attempt to measure separate modes of change. During Phases 1, 2, and 3, the change in node flow equality was + , - , and - , respectively (and opposite for hierarchy), while the change in redundancy was,++ , and - , respectively (and opposite for efficiency). Similar phase-defining differences in direction of change occurred for combinations of conditional entropy $(+,+,-)$ and mutual constraint $(-$, $-,+)$ for the open-model. Thus, the information theory approach does indeed capture structural changes in the U.S. economy.

In Phase 1, money flowed more equally both for the transactions among economic sectors and through each economic sector. In Phase 2, money began to flow less equally through each sector (e.g., total throughput). In Phase 3, money also began flowing in a less distributed manner among sectors. Thus, Phase 2 was a transition from a time where all indicators pointed to increasingly uniform distribution to one in which all indicators pointed to increasingly less uniform distribution. A research question remains as to whether this is a natural progression.

Second, increasing gross power input and net power ratio $\left(\mathrm{NPR}_{\text {economic }}\right)$ for the U.S. economy coincided with a trend toward more uniform distribution of monetary flows (increased conditional entropy and redundancy) and a decrease in efficient flow distribution (decreased mutual constraint and efficiency) among economic sectors of the U.S. economy. Because I model the economy as the same 37 sectors from 1947 to 2012, this increased distribution along with increased NPR economic also represents monetary flows going into new economic sectors that practically did not exist in the earlier years (e.g., "aircraft and parts" Sector 27 and "amusements" Sector 34).

Third, after 2002, when the NPR economic began to decrease and gross power input was approximately constant, the monetary flows of the U.S. economy became more concentrated as represented by both increases in node flow hierarchy, mutual constraint, and efficiency. Increased expenditures by energy and food sectors were the drivers of the post-2002 economy-wide structural change. Further, these increased expenditures are driven by the approach of biophysical constraints that are no longer allowing the production of ever-cheaper food and energy (King 2015b; King et al. 2015b). To be clear, energy and food intermediate expenditures are still a relatively small share compared to the 1950s, but they are no longer getting cheaper as in the past. Per Stein's Law, "If something cannot go on forever, it will stop" (Samuelson 2013). There are, of course, numerous studies projecting or searching for the signs of stagnation in growth or maturing of our society or economy, which is an expectation from a systems/ecosystems point of view that considers the finite nature of the Earth as a fundamental constraint (for some studies, see 
Meadows et al. (1972), Brown et al. (2011), Turchin and Nefedov (2009), Brown et al. (2014), DeLong and Burger (2015) and Matutinovií et al. (2016)).

Another way of summarizing these conclusions on the trends is that the U.S. economy transitioned from a relatively low metabolic (small low-consuming population) and resource-rich state (Phase 1) to one that has high metabolism (large high-consuming population) and is relatively resource-limited (Phase 3 ) in that it no longer can make energy and food cheaper. Since 2012, energy expenditures have dropped, particularly due to a decline in oil prices in 2015 (from relatively high prices, >90 2016/BBL, from 2012 to 2014). However, we must wait until the BEA produces the $2017 \mathrm{I}-\mathrm{O}$ tables to determine if the 2002-2012 trend continues.

There are competitive- and resource-based explanations for the phases I have outlined in the results. Phase 1 (from 1947 to 1967) was a period in which there were no apparent limits to U.S. economic expansion. The U.S. was the major market economy as Western Europe and Japan were rebuilding from World War II. Thus, the U.S. had little economic competition. Declining food expenditures (e.g., food = energy) along with cheap and abundant energy via prolific oil fields (e.g., in Texas) enabled the U.S. to control energy prices. Texas oil production actually had to be held back by the Texas Railroad Commission to prevent price collapse. During this time, the U.S. economy built up 'structural reserves' in the sense that money became more evenly spread throughout the domestic economy. By all measures in this paper, monetary flows became more evenly distributed during Phase 1.

The years 1967-2002 (Phase 2) are characterized by energy and environmental constraints affecting the U.S. economy for the first time. The Clean Air Act (1970) and Clean Water Act (1972) were substantially increased in scope and enforcement. Further, peak U.S. crude oil production in 1970 enabled the Arab oil embargo of 1973, and OPEC's increase in posted oil price in 1973 (Mitchell 2013), to raise oil prices to such a degree to cause major reactions by importing countries. The environmental and energy changes encouraged significant investment in utilities (e.g., wastewater treatment) and resource extraction (see Table 17) along with a focus on consumer energy efficiency for the first time since industrialization. As examples, the U.S. created car fuel efficiency standards known as Corporate Average Fuel Economy (CAFE), shifted away from using oil for power generation (and temporarily also away from natural gas and toward coal), and expanded oil production into Alaska and the Gulf of Mexico. These and other macroeconomic factors (increasing debt ratios non-financial corporations (Minsky 2016; pp. 48-52); the end of spending for the Vietnam War; and a transition to government deficits in 1970
(Galbraith 2014; p. 41) resulted in gross power consumption and GDP increasing at a slower rate than before the 1970s. Starting in 1972, the U.S. has no longer been a net exporter of goods and services (except for 1982), and the oil and gas sector has been a net importer (in monetary terms) since 1967 (see Figure S7).

Thus, economic transactions stopped becoming more evenly distributed as the annual changes in conditional entropy and indeterminacy were close to zero. Hierarchy started to increase as fast as it decreased during Phase 1 while redundancy kept increasing. This combination of metrics indicates that some sectors started attracting an increased share of total flows through them even while all flows within the economy became more distributed.

The years 2002-2012 (Phase 3) are unique in that the I$\mathrm{O}$ tables became more concentrated by all measures (the exact opposite trends of Phase 1). Mutual constraint, hierarchy (of node flows), and efficiency (of all flows) all increased during this time, while conditional entropy decreased. These changes are largely due to shifts toward increased energy ("Oil and Gas Extraction" Sector 3) and food sector purchases.

\section{Implications of Modeled Number of Sectors}

In each individual time series, I keep the number of sectors constant over all years. Thus, it is difficult to test the idea that more complexity is associated with "differentiation in structure (more parts to a system and, in particular, more kinds of parts)" (Tainter 2011). What can be said regarding the trends of modeling the economy with more sectors (Figs. 8, 9) is that representing the economy with more sectors mathematically produces higher hierarchy (lower equality) and efficiency (lower redundancy). That is to say, by modeling more sectors the same total system throughput can be distributed across a larger matrix. Thus, as the number of sectors increases, there are an increasing number matrix entries with smaller flows, and the mathematics inevitably produce higher hierarchy and efficiency for the same economy. The opposite trend happens modeling the economy with fewer sectors.

The mathematics of mutual constraint and conditional entropy, on the other hand, are such that the metrics do not change only by adding more sectors (or nodes) with zeros flows. For example, consider modeling an "internet" sector. In 1947, this sector would have no purchases or sales. Just by adding an internet sector (with zero sales and purchases) to the 1947 I-O table (e.g., before the internet), the absolute information theory metrics would not change, but equality and hierarchy would because they are defined relative to the size of the network. This is possibly an inherent advantage of applying information theory metrics to weighted networks in that zero entries have no impact on 
the absolute metric, or more generally, the larger flows have more impact on the calculations.

In considering the effect network size on aggregate metrics, there are possibly some insights to take from the ecological literature on the study of food webs. Bersier and Sugihara (1997) studied various food webs of different sizes and determined that there was a critical food web size. For analyzing individual species as nodes in the network, food webs smaller than 12 species indicated network metrics scaled with size, but those larger than 12 generally showed scale invariance (e.g., metrics did not change with more species defined in food webs) (Bersier and Sugihara 1997). When they alternatively decided to describe food webs by trophic types instead of individual species, they found the break point size was 7. It is a complete coincidence that I represented the $\mathrm{I}-\mathrm{O}$ tables as $12-$ and 7 -sector networks as I was unaware of this previous literature before performing my analysis.

One important difference between the I-O table constructions and those of food webs is explained by quoting (Bersier and Sugihara 1997): "The upper and lower limits of the properties, given that there are no loops of the kind where species A eats species A or species A eats species B and species B eats species A ..." The authors are explaining how certain values of their network metrics are limited by the constraints of constructing a viable food web. The I-O tables generally include the types of flows that they avoid. That is to say, economic sector A does purchase from and sell to Sector B and most sectors have considerable "cannibalistic" economic flows in which a sector purchases and sells to itself (e.g., transactions occur on the diagonal of the I-O matrix).

Thus, it is not clear whether research on directed graphs that describe ecological food chains is applicable for economic I-O tables. It is likely that, due to increased constraints on flows in directed networks, directed and undirected networks reside in distinct locations in the information theory phase space. For example, Ulanowicz finds that ecosystems tend to reside near efficiency $=a=$ 0.4 , whereas my 37-sector $\mathrm{I}-\mathrm{O}$ tables are in a range $0.10<a=$ efficiency $<0.21$. Analysis of the tables for 40 countries in the World Input-Output Database (19962011) also indicates efficiency $=a$ is between 0.12 and 0.17 (Bond 2015).

\section{Organization and the Energy-Complexity Spiral}

One motivation for this work was to relate metrics of the structure of the U.S. Use tables to energy consumption and costs. In effect, this partially tests Joseph Tainter's theory of the "energy-complexity" spiral, and there are some insights from the calculations of this paper.
The value of the approach in this paper is that it presents a rigorous quantitative framework for calculating metrics that characterize the structure of flows within a network. Despite the clear methods for calculating the metrics, the interpretations of any "complexity" calculations are much less clear partly due to the interpretive nature of a word such as "complexity." Most people would generally consider the U.S. economy as more complex today than in 1947-we have more technology and types of jobs (e.g., computers and information technology). But this general conclusion is not necessarily based on a structured framework.

Tainter suggests that higher complexity comes with an "increase in organization," where "organization is defined as constraints on behavior," and increased "economic differentiation." Using these definitions, an increase in organization can be indicated by increasing mutual constraint, hierarchy, and/or efficiency. None of these increase in Phase 1, a time of practically no economic or resource constraints. Only hierarchy increases in Phase 2, a time of increased resource constraints. All three "organization" metrics increase in Phase 3 (for closed and open-models), a time of increasing resource constraints (e.g., cost of food and energy) and economic competition (e.g., outsourcing to Asia) more so than the other phases.

Tainter (2011) also implies that societies with surplus energy (e.g., net energy), such as industrial society today, also increase complexity: "Abundant, inexpensive energy generates increasing complexity, and simultaneously produces new kinds of problems such as waste and climate change." If we take indeterminacy, or joint entropy, to be a measure of total system uncertainty and assume that higher uncertainty comes with more complex systems, then we can conclude that increased gross and net energy accompanied increased complexity of the U.S. economy during Phase 1 . The rationale here is that a more complex system is harder to understand, and thus, its internal flows have higher indeterminacy, or entropy. The corollary would be that the U.S. economy is decreased in complexity during Phase 3 because both open- and closed-model indeterminacy declined, along with gross power and net power ratio. The view of indeterminacy, or entropy, as a direct metric for complexity is perhaps the most consistent with Tainter's notion.

Sharing is another concept of Tainter's definition of complexity: that increased inequality is one measure of increased complexity. The Gini coefficient is often used a metric for inequality, and my measure of hierarchy is similar. After 1967 when quantifiable energy and economic constraints occurred, the hierarchy of the I-O tables increases as monetary flows are more concentrated through fewer sectors. The magnitude of the rate of change is 
higher for the closed versus open-model for Phases 1 and 3, but not Phase 2. The higher rate of Phase 2 increase in hierarchy for the open-model indicates that the input and output flows became concentrated more quickly than within the intermediate transactions of the $\mathrm{I}-\mathrm{O}$ table.

In summary, the calculations in this paper provide evidence that resource (e.g., energy) constraints influence the structure of the economy in some fundamental way. During Phase 1, we might say the U.S. became less complex as resources were abundant, cheap, and money became more evenly distributed (e.g., equality increased). However, we might say the economy became more complex if we use indeterminacy as a metric of complexity. During Phase 3, the opposite situation occurred, and we might say the economy became more complex by increasing hierarchy as organization in response to increased food and energy costs (e.g., constraints). On the other hand, Phase 3 can be interpreted as a time of decreasing complexity if indeterminacy is used as a metric for complexity. My conclusion is that complexity as a term is insufficient to characterize the changing flows in the U.S. I-O tables and likely any network.

I do not posit that my findings relate to personal social interaction. For example, some social and psychological literature investigates hierarchy and egalitarianism within teams, businesses, and communities. While it is commonly found that hierarchies emerge naturally to enable higher group productivity (e.g., higher sales, less time per task), these are usually investigations about individual people working together on common tasks (Ronay et al. 2012; Van Berkel et al. 2015). Interestingly, Van Berkel et al. (2015) suggest that more mental effort is required to better exhibit egalitarian values. Whether this increased effort translates to power input into the economy is a good question for future research. The results of this paper do show that higher rates of increase in U.S. power consumption were associated with increasing "egalitarianism" in terms of distribution of money in the I-O tables.

\section{Concluding Remarks}

In this paper, I sought evidence as to whether the U.S. economy has, over time (1) expended reserves to increase performance, (2) increased in complexity, and/or (3) increased in hierarchy in relation to total power consumption and/or the relative cost of energy and food to the U.S. economy. Overall, the analyses of this paper generally support the idea that the power and cost metrics coincide with a significant changes in the economic structure of the U.S. economy since 1947. One major structural shift occurred between 1967 and 1972, and the other around the turn of the twenty-first century.
Increasing rates of gross power consumption, as well as decreasing share of intermediate expenditures of the food and energy sectors, correlate with increased distribution of money among economic sectors, and vice versa. This result holds for both the closed (intermediate transactions only) and open (including value added as input and GDP as output) model representations of the economy. Thus, the U.S. economy did seem to expend structural reserves (e.g., decrease conditional entropy, redundancy, and equality) and increase efficiency (as well as mutual constraint and hierarchy) when food and energy expenditures increased after 2002.

Further, the findings of this paper are important for future economic modeling because they show how the level of detail within data and models affects system-wide distributional metrics of economic transactions. Thus, models of very simplified economies (e.g., 2-sector models) might not be able to describe enough detail or the correct trends.

Acknowledgments I thank Chester Polson for help on organization and interpretation of the underlying BEA input-output table data. I think Dr. Michael Blackhurst and Stephen Bond for intellectual discussions and guidance.

\section{Compliance with ethical standards}

Conflict of interest The author declares no conflict of interest. This research was performed without direct financial support. Dr. King's research funders are listed on his Web site at http://careyking.com/ projects/.

\section{References}

Ayres RU (2008) Sustainability economics: where do we stand? Ecol Econ 67(2):281

Ayres RU, Warr B (2005) Accounting for growth: the role of physical work. Struct Change Econ Dyn 16:181

Bersier LF, Sugihara G (1997) Scaling regions for food web properties. Proc Natl Acad Sci 94(4):1247. http://www.pnas. org/content/94/4/1247.abstract

Bond SR (2015) Relating energy use to economic complexity. Master's thesis, University of Texas at Austin

Brandt AR, Dale M, Barnhart CJ (2013) Calculating systems-scale energy efficiency and net energy returns: a bottom-up matrixbased approach. Energy 62:235. doi:10.1016/j.energy.2013.09. 054. http://www.sciencedirect.com/science/article/pii/S036054 4213008207

Brown JH, Burnside WR, Davidson AD, Delong JR, Dunn WC, Hamilton MJ, Mercado-Silva N, Nekola JC, Okie JG, Woodruff WH, Zuo W (2011) Energetic limits to economic growth. BioScience 61:19. doi:10.1525/bio.2011.61.1.7

Brown JH, Burger JR, Burnside WR, Chang M, Davidson AD, Fristoe TS, Hamilton MJ, Hammond ST, Kodric-Brown A, MercadoSilva N, Nekola JC, Okie JG (2014) Macroecology meets macroeconomics: resource scarcity and global sustainability. Ecol Eng 65:24. doi:10.1016/j.ecoleng.2013.07.071 
Bullard CW III, Herendeen RA (1975) The energy cost of goods and services. Energy Pol 3(4):268. doi:10.1016/0301-4215 (75) $90035-X$

Bureau of Economic Analysis (2016) Table 1.1.3. Real gross domestic product quantity indexes (A). http://www.bea.gov/ iTable/iTable.cfmReqID $=9 \&$ step $=1$ \#reqid $=9 \&$ step $=3 \&$ isuri $=1 \&$ $903=3$. Accessed 04 July 2016

Costanza R, Herendeen RA (1984) Embodied energy and economic value in the United States economy: 1963, 1967, and 1972. Resour Energy 6:129

DeLong JP, Burger O (2015) Socio-economic instability and the scaling of energy use with population size. PLoS One. doi:10. 1371/journal.pone.0130547. http://dx.doi.org/10.1371

Fouquet R (2008) Heat, power, and light: revolutions in energy services. Edward Elgar Publishing Limited, Northampton

Galbraith JK (2014) The end of normal: the great crisis and the future of growth. Simon \& Schuster, New York

Georgescu-Roegen N (1971) The entropy law and the economic process. Harvard University Press, Cambridge

Georgescu-Roegen N (1979) Energy analysis and economic valuation. South Econ J 45(4):1023

Goerner SJ, Lietaer B, Ulanowicz RE (2009) Quantifying economic sustainability: implications for free-enterprise theory, policy and practice. Econ Ecol 69(1):76

Hall CAS, Klitgaard KA (2012) Energy and the wealth of nations: understanding the biophysical economy, 1st edn. Springer, Berlin

Hall CAS, Cleveland CJ, Kaufmann RK (1986) Energy and resource quality: the ecology of the economic process. Wiley, New York

Hall CAS, Balogh S, Murphy DJR (2009) What is the minimum EROI that a sustainable society must have? Energies 2:25

Hidalgo CA, Hausmann R (2009) The building blocks of economic complexity. Proc Natl Acad Sci 106(26):10570. doi:10.1073/ pnas.0900943106. http://www.pnas.org/content/106/26/10570. abstract

Hirata H, Ulanowicz RE (1984) Information theoretical analysis of ecological networks. Int J Syst Sci 15(3):261

Ho MW, Ulanowicz R (2005) Sustainable systems as organisms? Biosystems 82(1):39

Huang J, Ulanowicz RE (2014) Ecological network analysis for economic systems: growth and development and implications for sustainable development. PLoS One 9(6):1. doi:10.1371/journal. pone. 0100923

Kalimeris P, Richardson C, Bithas K (2014) A meta-analysis investigation of the direction of the energy-GDP causal relationship: implications for the growth-degrowth dialogue. J Clean Prod 67:1

Kander A, Stern DI (2014) Economic growth and the transition from traditional to modern energy in Sweden. Energy Econ 46:56. doi:10.1016/j.eneco.2014.08.025. http://www.sciencedirect.com/ science/article/pii/S0140988314002072

Kharrazi A, Rovenskaya E, Fath BD, Yarime M, Kraines S (2013) Quantifying the sustainability of economic resource networks: an ecological information-based approach. Ecol Econ 90:177

King CW (2010) Energy intensity ratios as net energy measures of United States energy production and expenditures. Environ Res Lett 5:044006. http://stacks.iop.org/1748-9326/5/044006

King CW (2014) Matrix method for comparing system and individual energy return ratios when considering an energy transition. Energy 72:254. doi:10.1016/j.energy.2014.05.032. http://www. sciencedirect.com/science/article/pii/S0360544214005817

King CW (2015a) Comparing world economic and net energy metrics, part 3: macroeconomic historical and future perspectives. Energies 8(11):12348. doi:10.3390/en81112348. http:// www.mdpi.com/1996-1073/8/11/12348
King CW (2015b) The rising cost of resources and global indicators of change. Am Sci 103:6

King CW, Hall CAS (2011) Relating financial and energy return on investment. Sustainability 3(10): 1810

King CW, Maxwell JP, Donovan A (2015a) Comparing world economic and net energy metrics, part 1: single technology and commodity perspective. Energies 8(11):12346. doi:10.3390/ en81112346. http://www.mdpi.com/1996-1073/8/11/12346

King CW, Maxwell JP, Donovan A (2015b) Comparing world economic and net energy metrics, part 2: total economy expenditure perspective. Energies 8(11):12347. doi:10.3390/ en81112347. http://www.mdpi.com/1996-1073/8/11/12347

Kümmel R (2011) The second law of economics: energy, entropy, and the origins of wealth. Springer, Berlin

Lambert JG, Hall CA, Balogh S, Gupta A, Arnold M (2014) Energy, EROI and quality of life. Energy Pol 64:153. doi:10.1016/j. enpol.2013.07.001. http://www.sciencedirect.com/science/arti cle/pii/S0301421513006447

MacKay DJC (2003) Information theory, inference, and learning algorithms. Cambridge University Press, Cambridge. http:// www.inference.phy.cam.ac.uk/itprnn/book.pdf

Matutinovií I, Salthe SN, Ulanowicz RE (2016) The mature stage of capitalist development: models, signs and policy implications. Struct Change Econ Dyn 39:17

McNerney J, Fath BD, Silverberg G (2013) Network structure of inter-industry flows. Phys A: Stat Mech Appl 392(24):6427. doi:10.1016/j.physa.2013.07.063. http://www.sciencedirect.com/ science/article/pii/S0378437113006948

Meadows DH, Meadows DL, Randers J, Behrens WWI (1972) Limits to growth: a report for the club of Rome's project on the predicament of mankind. Universe Books, New York

Miller RE, Blair PD (2009) Input-output analysis: foundations and extensions. Cambridge University Press, Cambridge

Minsky H (2016) Can it happen again?. Routledge, London

Mitchell T (2013) Carbon democracy: political power in the age of oil. Verso, London

Modahl IS, Raadal HL, Gagnon L, Bakken TH (2013) How methodological issues affect the energy indicator results for different electricity generation technologies . Energy Policy. doi:10.1016/j.enpol.2013.09.005. http://www.sciencedirect.com/ science/article/pii/S0301421513009294

Mulder K, Hagens NJ (2008) Energy return on investment: toward a consistent framework. Ambio 37(2):74

Murphy DJR, Hall CAS, Cleveland CJ (2011) Order from chaos: a preliminary protocol for determining EROI for fuels. Sustainability 3(10): 1888

Odum HT (1971) Environment, power, and society. Wiley, New York

Ronay R, Greenaway K, Anicich EM, Galinsky AD (2012) The path to glory is paved with hierarchy: when hierarchical differentiation increases group effectiveness. Psychol Sci 23(6):669. doi:10.1177/0956797611433876. http://pss.sagepub.com/con tent/23/6/669.abstract

Samuelson RJ (2013) Is Steins law real?. Washington Post . http:// www.washingtonpost.com/opinions/robert-j-samuelson-issteins-law-real/2013/05/30/716942f2-c942-11e2-8da7-d274 bc611a47_story.html

Santos J, Domingos T, Sousa T, Aubyn MS (2016) Does a small cost share reflect a negligible role for energy in economic production? Testing for aggregate production functions including capital, labor, and useful exergy through a cointegrationbased method, MPRA Paper No. 70850. Munich Personal RePEc Archive, pp 1-61. https://mpra.ub.uni-muenchen.de/ $70850 /$

Shannon C, Weaver W (1962) The mathematical theory of communication. University of Illinois Press, Urbana 
Smil V (2008) Energy in nature and society: general energetics of complex systems. The MIT Press, Cambridge

Solow RM (1956) A contribution to the theory of economic-growth. Q J Econ 70(1):65. doi:10.2307/1884513

Stern DI, Kander A (2012) The role of energy in the industrial revolution and modern economic growth. Energy J. doi:10.5547/ 01956574.33.3.5

Tainter J (1988) The collapse of complex societies. cambridge University Press, Cambridge

Tainter JA (2011) Energy, complexity, and sustainability: a historical perspective. Environ Innov Soc Transit 1:89

Turchin P, Nefedov SA (2009) Secular cycles. Princeton University Press, Princeton

Ulanowicz RE (2002) The balance between adaptability and adaptation. Biosystems 64(1-3):13

Ulanowicz RE (2004) Quantitative methods for ecological network analysis. Comput Biol Chem 28:321

Ulanowicz RE (2009) The dual nature of ecosystem dynamics. Ecol Model 220(16):1886. doi:10.1016/j.ecolmodel.2009.04.015. http://www.sciencedirect.com/science/article/pii/S03043800090 02695
Ulanowicz RE, Hannon BM (1987) Life and the production of entropy. Proc R Soc Lond. Ser B. Biol Sci 232(1267):181. doi:10.1098/rspb.1987.0067. http://rspb.royalsocietypublishing. org/content/232/1267/181.abstract

Ulanowicz RE, Wolff WF (1991) Ecosystem flow networks: loaded dice? Math Biosci 103:45

Ulanowicz RE, Goerner SJ, Lietaer B, Gomez R (2009) Quantifying sustainability: resilience, efficiency and the return of information theory. Ecol Complex 6(1):27

Van Berkel L, Crandall CS, Eidelman S, Blanchar JC (2015) Hierarchy, dominance, and deliberation: egalitarian values require mental effort. Personal Soc Psychol Bull 41(9):1207. doi:10.1177/0146167215591961. http://psp.sagepub.com/con tent/41/9/1207.abstract

WE Council (2013) World energy resources, 2013 survey: summary. Tech. rep

Zorach AC, Ulanowicz RE (2003) Quantifying the complexity of flow networks: how many roles are there? Complexity 8(3):68 